Document downloaded from:

http://hdl.handle.net/10251/64816

This paper must be cited as:

Filgueiras-Rama, D.; Calvo Saiz, CJ.; Salvador-Montañés, O.; Cádenas, R.; Ruiz-Cantador, J.; Armada, E.; Rey, JR.... (2015). Spectral analysis-based risk score enables early prediction of mortality and cerebral performance in patients undergoing therapeutic hypothermia for ventricular fibrillation and comatose status. International Journal of Cardiology. 186:250-258. doi:10.1016/j.ijcard.2015.03.074.

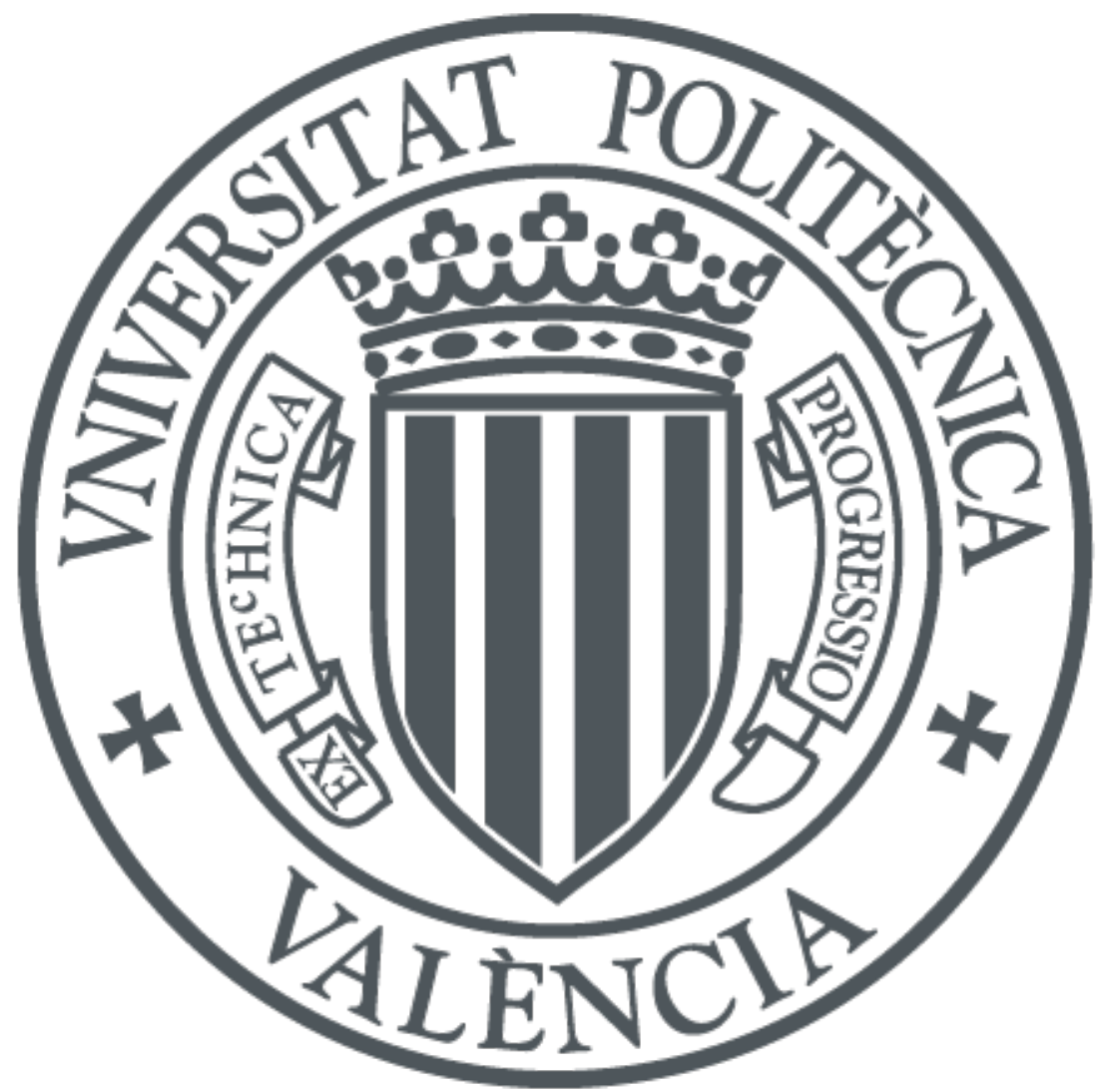

The final publication is available at

http://dx.doi.org/10.1016/j.ijcard.2015.03.074

Copyright Elsevier

Additional Information 


\section{Spectral Analysis-Based Risk Score Enables Early Prediction of Mortality and Cerebral Performance in Patients Undergoing Therapeutic Hypothermia for Ventricular Fibrillation and Comatose Status.}

David Filgueiras-Rama*,a,c,d,1,2 , Conrado J. Calvo ${ }^{\text {b,e,2,3, Óscar Salvador-Montañés }}{ }^{\mathrm{d}, 3,4}$, Rosalía Cádenas $^{\mathrm{d}, 4}$, Jose Ruiz-Cantador ${ }^{\mathrm{d}, 4}$, Eduardo Armada ${ }^{\mathrm{d}, 4}$, Juan Ramón Rey ${ }^{\mathrm{d}, 4}$, JL Merino $^{\mathrm{d}, 4}$, Rafael Peinado ${ }^{\mathrm{d}, 4}$, Nicasio Pérez-Castellano ${ }^{\mathrm{c}, 3}$, Julián Pérez-Villacastín ${ }^{\mathrm{c}, 3}$, Jorge G. Quintanilla ${ }^{\mathrm{a}, 3}$, Santiago Jiménez ${ }^{\mathrm{b}, 3}$, Francisco Castells $\mathrm{s}^{\mathrm{b}, \mathrm{e}, 3}$, Francisco J. Chorro ${ }^{\mathrm{e}, 3}$, JL López-Sendón $^{\mathrm{d}, 3}$, Omer Berenfeld ${ }^{\mathrm{f}, 3}$, José Jalife ${ }^{\mathrm{a}, \mathrm{f}, 3}$, Esteban López de Sá ${ }^{\mathrm{d}, 3,4,5}$, José Millet $^{\mathrm{b}, \mathrm{e}, 3,5}$.

${ }^{a}$ Atherothrombosis, Imaging and Epidemiology Department. Fundación Centro Nacional de Investigaciones Cardiovasculares (CNIC), Madrid, Spain.

b Bio-ITACA Grupo de Bioingeniería, Instituto de Aplicaciones Avanzadas, Dpto Ingeniería Electrónica, Universitat Politècnica de Valencia, Spain

${ }^{\mathbf{c}}$ Hospital Clínico San Carlos, Madrid, Spain.

${ }^{\text {d }}$ Hospital Universitario La Paz, IdiPaz, Madrid, Spain.

${ }^{\text {e }}$ Grupo Electrofisiología y Bioingeniería, Microcluster en Protección Cardiovascular, Dpto de Medicina, Fundación INCLIVA Hospital Clínico, Valencia, Spain

${ }^{\mathbf{f}}$ Center for Arrhythmia Research. Cardiovascular Research Center, Department of Internal Medicine, University of Michigan, Ann Arbor, USA.

${ }^{1}$ This author designed and supervised the study, takes responsibility for all aspects of the reliability and freedom from bias of the data presented and their discussed interpretation.

${ }^{2}$ Both authors contribute equally to this manuscript. 
3 This author takes responsibility for all aspects of the reliability and freedom from bias of the data presented and their discussed interpretation.

${ }^{4}$ This author contributed to data collection, review and interpretation.

${ }^{5}$ Both authors contribute as senior authors.

* Corresponding author at: Centro Nacional de Investigaciones Cardiovasculares (CNIC). Melchor Fernández Almagro, 3. 28029. Madrid. Spain. Phone: +34-914531200. Ext.1510. Fax:+34-91-4531265. Email address: david.filgueiras@cnic.es

Grant Support: The CNIC is supported by the Spanish Ministry of Economy and Competitiveness and the Pro-CNIC Foundation.

Disclosures: None.

Keywords: Cardiac arrest; cerebral injury; early prognosis; ventricular fibrillation; dominant frequency.
Abbreviations (not standard in this field)
$\mathrm{FNP}=$ Favorable neurological performance
HL-PSDR = High-to-low power spectral density ratio
HL-pKR = High-to-low peak ratio 


\begin{abstract}
Background: Early prognosis in comatose survivors after cardiac arrest due to ventricular fibrillation (VF) is unreliable, especially in patients undergoing mild hypothermia. We aimed at developing a reliable risk-score to enable early prediction of cerebral performance and survival.
\end{abstract}

Methods: Sixty-one out of 239 consecutive patients undergoing mild hypothermia after cardiac arrest, with eventual return of spontaneous circulation (ROSC), and a comatose status on admission fulfilled the inclusion criteria. Background clinical variables, VF time and frequency domain fundamental variables were considered. The primary and secondary outcomes were a favorable neurological performance (FNP) during hospitalization and survival to hospital discharge, respectively. The predictive model was developed in a retrospective cohort $(n=32$; September 2006-September 2011, 48.5 \pm 10.5 months of follow-

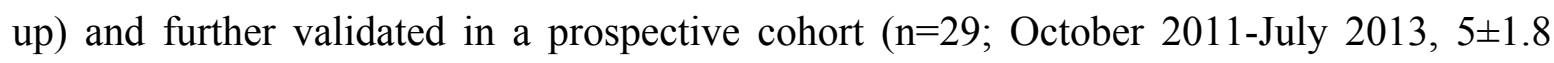
months of follow-up).

Results: FNP was present in $16(50.0 \%)$ and 21 patients $(72.4 \%)$ in the retrospective and prospective cohorts, respectively. Seventeen (53.1\%) and 21 patients (72.4\%), respectively, survived to hospital discharge. Both outcomes were significantly associated $(\mathrm{p}<0.001)$. Retrospective multivariate analysis provided a prediction model (sensitivity=0.94, specificity $=1$ ) that included spectral dominant frequency, derived power density and peak ratios between high and low frequency bands, and the number of shocks delivered before ROSC. Validation on the prospective cohort showed sensitivity $=0.88$ and specificity $=0.91$. A model-derived risk-score properly predicted $93 \%$ of FNP. Testing the model on followup showed a c-statistic $\geq 0.89$. 
Conclusions: A spectral analysis-based model reliably correlates time-dependent VF spectral changes with acute cerebral injury in comatose survivors undergoing mild hypothermia after cardiac arrest. 


\section{Introduction.}

Both in-hospital and out-of-hospital cardiac arrest due to ventricular fibrillation (VF) are associated with high mortality rates and significant cerebral disability.[1, 2] VF-derived cerebral injury is a very sensitive time-dependent condition with dramatic social and personal consequences. The absence of cerebral blood flow during VF leads to ischemic damage within a few minutes, which increases after reperfusion due to generation of oxygen free radicals and activation of degradation enzymes, together with other mediators.[3] To date, mild hypothermia is the only therapy that has shown to increase survival rates and functional outcomes in comatose survivors of cardiac arrest due to VF, [46] even though cardiopulmonary resuscitation (CPR) technique and some specific drugs are considered influential during resuscitation. However, the use of sedative and neuromuscular blocking drugs in cooled patients may mask neurological damage and delay examination. Furthermore, early prognosis within the first $72 \mathrm{~h}$ after cardiac arrest remains unreliable, which is especially relevant in those patients undergoing highly specialized intensive care who might not have any hopes for recovery.

Reducing the time to DC shock after VF onset is vital to restore spontaneous circulation and minimize cerebral injury.[7] However, the exact time in VF is difficult to determine even after witnessed cardiac arrest. Many VF episodes may initiate as ventricular tachycardia and cerebral blood flow might still persist until VF develops.[8] Reliable experimental data from waveform analysis during VF indicate that both spectral dominant frequency (DF) and median frequency decrease after onset of VF.[9, 10] In the clinical setting, such a decrease in spectral values correlates with poor defibrillation success and no return of spontaneous circulation (ROSC).[11] Moreover, retrospective data in patients with out-of-hospital cardiac arrest and VF have shown that a $5.61 \mathrm{~Hz}$ DF threshold can serve as a good predictor for 1-year survival after discharge.[12] Therefore, we hypothesize that spectral analysis of 
VF before a DC shock will accurately reflect the degree of acute cerebral injury as a consequence of time in VF and concomitant myocardial ischemia.

Here, we analyzed VF waveform properties before the first DC shock in patients undergoing therapeutic hypothermia due to comatose status after advanced life support (ALS) and ROSC. We aimed to identify spectral parameters that in combination with clinical variables may serve to develop a reliable model and risk score to enable early prediction of cerebral performance and survival to hospital discharge. We also studied the capacity of the model to predict both outcomes at follow-up.

\section{Methods.}

\subsection{Study design.}

The study was performed in a referral center for out-of-hospital cardiac arrest (Hospital Universitario La Paz, Madrid, Spain), in which mild therapeutic hypothermia is routinely used in comatose survivors after the event. The emergency service and ambulances in the region have trained medical staff and nurses, all coordinated by a central station to minimize both time to ALS and transportation to a referral center. The study included consecutive patients who underwent mild hypothermia after cardiac arrest due to VF, eventually with ROSC, and comatose status (Glasgow Coma Scale $\leq 8$ ) on admission. Patients with witnessed or un-witnessed documented VF were eligible for the study, as long as VF traces before the first DC shock had enough quality and duration ( $\geq 3 \mathrm{~s}$ ) for digitization and analysis of spectral parameters, respectively. We excluded patients with early mortality or hemodynamic instability leading to incomplete 24-h of mild hypothermia, and absence of subsequent withdrawal of sedation to assess cerebral performance. Other exclusion criteria were age $<18$ years, Glasgow Coma Scale score after ROSC $>8$, nonshockable or shockable rhythms other than VF, a terminal illness or cognitive deterioration 
present before the cardiac arrest, and possible causes of coma other than cardiac arrest. The study was divided in two groups, as follows: Group 1 with eligible patients from September 2006 to September 2011 and retrospective data analysis, and Group 2 with eligible patients from October 2011 to July 2013, in whom we prospectively studied the utility of the predictive algorithm developed in Group 1. All data were collected from a prospective registry. The institutional ethics review committee approved prospective analysis of the patients, in accordance with the ethical guidelines of the 1975 Declaration of Helsinki and European guidelines for good clinical practice.

\subsection{Hypothermia protocol.}

Patients admitted to the acute cardiac care unit (ACCU) underwent routine neurological evaluation before sedation, drug-induced paralysis and initiation of hypothermia protocol as described elsewhere.[6] Briefly, cooling with intravenous cold saline $\left(<8^{\circ} \mathrm{C}\right)$ was initiated on admission. This was followed by direct cooling of the blood using the Icy catheter (ZOLL Medical Corporation, Chelmsford, MA) positioned at the level of the inferior vena cava through the femoral vein. Cooling was set at a maximum rate with a target temperature either at 32,33 or $34^{\circ} \mathrm{C}$, which was maintained during 24 hours. Rewarming was controlled at a set rate of 0.1 to $0.3^{\circ} \mathrm{C}$ per hour to reach $37^{\circ} \mathrm{C}$ in 12 to 24 hours. Mechanical ventilation was adjusted to ensure normoxemia and normocapnia. Mean blood pressure was maintained between 85 and $100 \mathrm{~mm} \mathrm{Hg}$. Blood glucose level was ensured $<10 \mathrm{mmol} / \mathrm{l}$. Limitation of active ALS was considered in patients who remained deeply comatose after 5 days of evolution, as long as it was possible to reach an agreement with their representatives.

\subsection{Spectral Analysis.}

For each patient, we analyzed VF epochs prior to the first DC shock. Digitization was performed using a supervised semi-automatic approach based on region of interest selection, 
histogram thresholding and intensity transformations. Up to 5-s long segments were extracted after segmentation and signal codification from artifact-free VF tracings. Signals were band-pass filtered between 1.5 and $40 \mathrm{~Hz}$. Quality of extraction was visually inspected by two independent investigators. Averaged power spectral density was obtained at each frequency using the non-parametric Welch method for using fast Fourier transform and normalized to the peak power in the $1.5-10 \mathrm{~Hz}$ band for each patient. Both time and frequency domain variables were quantified across patients. Those included VF amplitude over time, amplitude spectral area (AMSA), DF, median frequency, approximate entropy regularity index, spectral regularity index, 1-Hz DF spectral concentration and normalized $80 \%$ power spectral density (see Figure 1 and Supplemental Methods for details). Investigators blinded to clinical outcome performed all data analysis, extraction and quantification using custom-made scripts of MATLAB software (V. 2010b, The Mathworks Inc, Natick, MA).

\subsection{Outcome.}

The primary outcome was a favorable neurological performance (FNP) during hospitalization. All patients were classified using the Pittsburgh outcome categorization of brain injury as follows: cerebral performance categories (CPC) 1 and 2 (good and moderate disability, respectively) were considered as FNP, and CPC 3, 4 and 5 (severe disability, vegetative state and brain death, respectively) were considered as a non-FNP (Supplemental Table 1).[13] Neurological outcome was established after in-hospital stabilization or before hospital discharge. In patients from group 1, retrospective data were obtained from clinical records during hospitalization.

The secondary outcome measure was survival to hospital discharge.

\subsection{Follow-up.}


Neurological outcome was prospectively assessed by in-person interview in all survivors after October 2011, either from group 1 or group 2. Specifically, patients from group 2 were evaluated between 3 and 6 months after hospitalization. Neurological outcome was also determined in both groups using the mini-mental state examination as follows: any score $\geq 24$ points (out of 30 ) indicated a good cognition. Scores $<24$ indicated cognitive impairment.[14] Only patients with both $\mathrm{CPC} \leq 2$ and mini-mental state examination score $\geq 24$ were considered to have FNP at follow-up.

Survival after hospitalization was assessed in group 1 after October 2011. In patients from group 2, survival was assessed at 6 months after hospital discharge.

\subsection{Statistical Analysis.}

All values are presented as median \pm SEM $\left(25^{\text {th }}, 75^{\text {th }}\right.$ percentiles $)$ except where noted. The retrospective cohort was used to develop a model for predicting the primary outcome. Each of the clinical, spectral and time domain VF variables underwent univariate analysis to evaluate its association with in-hospital FNP. Normal distribution of variables was assessed with Shapiro-Wilk test. Statistical significance was assessed by the T-test or the MannWhitney-Wilcoxon test, as appropriate. If necessary, we used Bonferroni correction for multiple comparisons. Categorical variables and percentile comparisons were compared using a Chi-squared test or the Fisher Exact test, as appropriate. $\mathrm{P}<0.05$ was considered statistically significant. Non-correlated variables, amongst statistically significant ones (Supplemental Figure 1), and clinical relevant variables were regressed out against the primary outcome by using a stepwise backward multivariate logistic regression approach. We aimed at predicting in-hospital FNP with the highest sensitivity and specificity achievable using the minimum number of variables. We validated the predictive accuracy of the model in the prospective cohort and tested the model during follow-up. We also 
studied in both groups the accuracy of the model in predicting survival. Patients from both groups were categorized according to their risk scores obtained in the multivariate analysis. Goodness of fit was assessed through Pearson residuals and Chi-squared deviance. To correct for bias, we obtained bootstrapped standard errors for weights. To guarantee robustness we used the Jackknife fitted regression weights to confirm the minimum mean squared error (See Supplemental Methods for details). All analyses were done using SSPS v21 and custom Matlab scripts for mathematical assistance.

\section{Results.}

The workflow of the study is depicted in Figure 2. A total of 239 patients undergoing mild hypothermia $(n=116$, retrospective cohort and $n=123$, prospective cohort) were assessed for eligibility during the study period. Sixty-one patients $(n=31$, group 1 and $n=29$, group 2$)$ fulfilled the inclusion criteria. The vast majority of patients were included after out-ofhospital cardiac arrest $(n=57)$. However, two patients in each group were included after inhospital cardiac arrest due to $\mathrm{VF}$, since comatose status was present after DC shock and ROSC. Baseline clinical characteristics and background treatment of both groups are shown in Table 1. Female sex, family history of sudden cardiac death and younger age were more frequent in group 2. Overall, the main cause of VF was coronary heart disease, either acute coronary syndromes $(n=27,45 \%)$ or chronic coronary disease $(n=14,23 \%)$, followed by idiopathic VF $(n=6,10 \%)$ and dilated cardiomyopathy $(n=6,10 \%)$ (Supplemental Figure 2).

\subsection{Outcomes.}

Primary and secondary outcomes are shown in Table 2 . Sixteen patients in group $1(50.0 \%)$ and 21 patients in group $2(72.4 \%)$ achieved FNP during hospitalization. Seventeen patients in group $1(53.1 \%)$ and 21 patients in group $2(72.4 \%)$ survived to hospital discharge. After a median follow-up of $48.5 \pm 10.5$ months $(7.0,68.7)$ in group 1,16 patients $(50.0 \%)$ were 
still alive, albeit $15(48.8 \%)$ showed FNP. In group 2, 20 patients $(68.8 \%)$ were alive at 6 months after hospital discharge and 19 (65.5\%) showed FNP after $5 \pm 1.8$ months $(3.5,7.5)$. Hospitalization outcomes were not statistically different between groups (Table 2). No patients were missed during follow-up. Outcomes and follow-up of each individual patient are depicted in Supplemental Figure 3. Four patients died (6.5\%) despite FNP. There was a statistically significant association between FNP and survival (Supplemental Table 2) in both groups $(\mathrm{p}<0.001)$.

\subsection{Prediction model.}

In creating the model we only considered the primary endpoint since mortality may occur in patients with FNP due to other causes non-directly related with cardiac arrest injury. The interval to ALS and total time of ALS reached statistical significance among clinical variables. All fundamental spectral and time domain VF variables, but VF signal amplitude, were significantly associated to the primary endpoint $(\mathrm{p}<0.05)$ (Table 3$)$. Interestingly, DF was strongly associated with FNP, which was reflected by the best univariate independent predictive accuracy in the retrospective and prospective cohorts (average 0.884) (Table 3). A cut-off at $3.9 \mathrm{~Hz}$ showed the highest sensitivity (0.88) and specificity (0.94) in predicting the primary endpoint in the retrospective cohort. Therefore, we used such a cut-off value to obtain two derived, also significant ( $<<0.001$. Table 3 ), spectral variables as follows: highto-low power spectral density ratio (HL-PSDR), as the relative power between high (3.9-10 $\mathrm{Hz})$ and low (1.5-3.9 Hz) bands, and high-to-low peak ratio (HL-pKR), as the relative number of spectral peaks above and below $3.9 \mathrm{~Hz}$ with power above $40 \%$ of the DF (Figure 1). Graphic representation of individual spectra and DF peaks of the entire population are shown in Figure 3A. The vast majority of patients with FNP during hospitalization showed DF values above $3.9 \mathrm{~Hz}$ (Figure 3A1), unlike those individuals with non-FNP, who had DF values below $3.9 \mathrm{~Hz}$ (Figure 3A2). Such differences were statistically significant both in the 
retrospective and prospective cohorts, as well as in the entire population $(\mathrm{p}<0.001$. Figure 3B). Moreover, DF values also showed significant differences between patients who survived and those who did not survive to hospital discharge $(\mathrm{p}<0.001$. Figure $3 \mathrm{~B})$.

Multivariate analysis identified DF, HL-pKR, HL-PSDR and the number of shocks delivered before ROSC as the best performance model to predict in-hospital FNP. Multivariate adjusted odds ratios are shown in Table 4. For the primary endpoint, the model achieved sensitivity $=0.94$ and specificity $=1(\mathrm{c}-$ statistic $=0.98)$. Validation on the prospective cohort also showed high sensitivity $(0.88)$ and specificity (0.91) (c-statistic=0.89). The multivariate model achieved sensitivity $=0.94$ and specificity $=0.94$ to predict in-hospital survival in the retrospective cohort (c-statistic $=0.95)$. For the secondary endpoint, predictive performance was also high in the prospective group (sensitivity $=0.88$, specificity $=0.91$, cstatistic $=0.92)$. ROC curves of the multivariate model are shown in Supplemental Figure 4. In-hospital performance of the model is shown in Table 5. Performance of the model for both outcomes at follow-up also reached high sensitivity, specificity and c-Statistic values (Supplemental Table 3).

\subsection{Risk score based on the predictive performance of the model.}

We used the best performance threshold obtained by the prediction model to define four risk subsets of the population for non-FNP, as follows: very low and low risk of non-FNP (expected FNP), high and very high risk of non-FNP (expected non-FNP). Interquartile ranges of individual variables within each of the risk score groups are shown in Supplemental Table 4. Figure 4A shows the observed and predicted probability of inhospital FNP for the entire population. The risk score correctly classifies more than $93 \%$ of observations. Only 1 patient in the retrospective cohort was classified as FNP, although the patient was non-FNP during hospitalization (False negative. Figure 4B1). Three patients in 
the prospective cohort were identified as false negative and false positives ( 1 and 2 patients, respectively). The risk score was very reliable in predicting neurological performance in the subgroups of very low and very high risk of non-FNP (Figure 4, B1 and B2). Multivariate adjusted logistic regression weights and statistics for each of the four variables included in the risk score are shown in Supplemental Table 5.

To test the reproducibility of the spectral variables present in the risk score, we quantified changes in the spectral components of VF prior the first reported DC shock using a 3-s sliding window shifted 0.2 -s from the DC shock. Interestingly, spontaneous variability of the spectral components a few seconds (maximum available=12 s) before the DC shock did not reflect significant changes in neurological performance prediction and risk score classification for the entire population (Supplemental Figure 5).

Further risk score validation in patients without comatose status after DC shock who did not undergo hypothermia $(\mathrm{N}=11)$, showed that risk score stratification properly provided FNP values in all cases, as expected (Supplemental Figure 6).

\subsection{Contribution of spectral parameters to the predictive performance of the model.}

The predictive performance of our model was highly dependent on incorporating the spectral variables. Thus, only considering the spectral parameters, the prediction model achieved high sensitivity (0.88) and specificity (0.86) in validation (c-statistic=0.88), compared with sensitivity $=0.62$ and specificity $=0.66(\mathrm{c}$-statistic $=0.67)$ using the number of shocks delivered before ROSC alone. Moreover, the best clinical prediction model using the most influential clinical factors in the univariate analysis (Table 3), we obtained a 5variable model that achieved sensitivity $=0.50$ and specificity $=0.71(\mathrm{c}$-statistic $=0.69)$ in the prospective cohort. The inclusion of additional clinical-relevant variables to achieve better 
prediction in training resulted in further decrease in the predictive performance of the model in the prospective cohort $(\mathrm{c}$-statistic $=0.63)$. Overall, clinical models did not achieve cstatistic values greater than 0.69 in the prospective cohort (Supplemental Table 6 and Supplemental Methods), which highlights the objective and reliable significance of spectral parameters.

\section{Discussion.}

We have introduced a novel practical approach aimed at predicting neurological performance and survival in patients undergoing therapeutic hypothermia after cardiac arrest due to VF and comatose status on admission. In brief, DF before the first DC shock is a strong independent predictor for both FNP and survival using a cut-off value of $3.9 \mathrm{~Hz}$. To increase the predictive accuracy, multivariate analysis identified DF, HL-pKR, HLPSDR and the number of shocks delivered before ROSC as the best performance model to predict both primary and secondary outcomes. The model showed sensitivity and specificity values above 0.88 and 0.91 in the validation prospective cohort. We also developed a risk score that properly predicted $93 \%$ of the in-hospital neurological outcome observed in the entire cohort.

Currently, the reliability of early prognosis in comatose survivors after cardiac arrest due to VF is very limited, which severely impairs the ability of physicians to provide accurate information to patients' relatives and to optimize the use of intensive-resource care. Standardization of mild hypothermia delays neurological evaluation and prognostication due to sedation as well as higher rates of misleading biomarker values within the first 24-48 h.[15] Moreover, the large variability of threshold biomarker values used to predict poor outcome and different measurement techniques makes it necessary to exert caution and question the prognostic accuracy provided by biochemical markers. 
Clinical variables are also inconsistent in their ability to predict both survival and neurological performance,[16] as we also showed after developing and validating the best clinical prediction models. Advanced age seems to be associated with decreased survival after cardiac arrest and resuscitation.[17] Interestingly, old age is not associated with nonFNP,[7] which supports the role of early and appropriate resuscitation to prevent cerebral injury.[18] However, FNP does not prevent later complications that may lead to in-hospital mortality, especially in old patients. Univariate analysis of our retrospective cohort also showed younger age as significantly associated with survival (data not shown). Conversely, age was not significantly associated with FNP. Likewise, inclusion of clinically relevant variables in the multivariate analysis did not result in age as a variable present in the predictive model.

Time to CPR after collapse has been shown to correlate with functional outcome.[7] Moreover, when performed properly, CPR improves functional outcome.[18] However, the quality of CPR administered by a bystander might be extremely variable even if performed by trained personnel,[18, 19] which might not add significant improvement in outcome.[4] The strong predictive value of DF and derived spectral variables may be explained by their ability to provide reliable information of both the time from VF onset and the quality of CPR. Thus, as shown in both humans and animal models, as the VF episode evolves, progressive myocardial ischemia leads to gradual decline in DF values.[9, 10, 20, 21] Stewart AJ et al. have shown that DF values fall with increased duration of collapse from $5.5 \mathrm{~Hz}$ at $3 \mathrm{~min}$ to a mean of $2.1 \mathrm{~Hz}$ at $20 \mathrm{~min}$.[10] Patients with a mean DF of $3.89 \pm 0.25$ $\mathrm{Hz}$ did not survive longer than $6 \mathrm{~h}$ after resuscitation, unlike patients with a mean DF of $5.60 \pm 0.25 \mathrm{~Hz}$ who did survive.[10] Similar DF values $(5.61 \mathrm{~Hz})$ have been reported by Goto $\mathrm{Y}$ et al. to predict 1-year survival in a retrospective cohort of patients after out-of- 
hospital cardiac arrest, although data about neurological performance were missed.[12] Our risk score identifies DF of $5.6 \pm 0.53 \mathrm{~Hz}$ and $6.35 \pm 0.75 \mathrm{~Hz}$ at low and very low risk of nonFNP, respectively.

The latter does not necessarily correlates with survival, which is reflected by the fact that 2 patients during hospitalization and another 2 patients during follow-up died despite FNP. Previous series have shown that the longer the time between CPR and ROSC the lower the survival rate[1,7], which is similar to what we observed in both cases with FNP and in-hospital mortality after the hypothermia protocol, in whom long CPR (20 and $42 \mathrm{~min}$ ) and high number of shocks (4 and 5) before ROSC were present. A significantly higher number of shocks delivered before ROSC was also present in patients with $\mathrm{DF}<3.9 \mathrm{~Hz}$ (Supplemental Figure 7), which highlights the difficulties of the heart for acute recovery.

\section{Mortality}

may also occur during the hypothermia protocol before withdrawing sedation. Thus, data from 4 out of 5 patients, who were excluded due to early mortality and impossibility to assess the primary outcome, showed that risk score stratification would have predicted FNP in 3 out of 4 (Supplemental Table 7). However, early mortality due to other medical circumstances did not allow recovery and neurological assessment.

Interestingly, CPR may increase DF values while coronary blood flow rises, [21, 22] which is also associated with increased probability of successful rescue shocks.[23] Increase in DF during resuscitation may explain false positive cases to predict FNP when CPR is delayed after collapse and DF is already low. The latter is supported by recent data by Freese et al. in patients with out-of-hospital cardiac arrest due to VF.[24] The authors showed that a waveform analysis algorithm to decide whether to apply an immediate defibrillatory shock or a CPR interval before the shock, did not improve overall survival to hospital discharge 
compared with a standard shock-first protocol. In the study, prognosis may have been determined by the spectral values at the beginning of CPR. Yet, an increase in favorable VF waveform parameters during CPR increases the probability of ROSC and survival to admission.[24]

DF alone showed the best univariate independent predictive accuracy amongst spectral variables. However, relying on DF alone may not be accurate in some cases; for instance when the DF peak is close to the cut-off value. Our multivariate model includes 2 additional derived measures (HL-PSDR and HL-pKR) that aided to clarify such cases. The probability of a favorable outcome increases as the relative power of high spectral bands $(3.9-10 \mathrm{~Hz})$ and the number of significant spectral peaks above $3.9 \mathrm{~Hz}$ also increase (Supplemental Figure 8). Recent data by Schoene et al. also highlights the role of AMSA over the course of the first 3 shocks during resuscitation in predicting survival and FNP in a large retrospective cohort of patients with out-of-hospital cardiac arrest due to VF.[25] Calculation of AMSA will typically provide higher AMSA values in traces with more highfrequency content, which agrees with our results based on DF. The data is also consistent with However, Schoene et al. did not distinguish between patients with or without comatose status on admission. Moreover, the authors did not provide information about post-cardiac arrest care using mild hypothermia. Here, we focused on a population with baseline comatose status on admission and mild hypothermia as uniform therapy to minimize postcardiac arrest syndrome. 
Finally, recent data prompted us to consider the role of hypothermia after cardiac arrest and whether a target temperate rather than controlling the body temperature at $36{ }^{\circ} \mathrm{C}$ must be pursued.[26] We speculate that temperature may have a minimum role in patients with very low risk (i.e., favorable risk score) or very high risk of cerebral injury at baseline (i.e., asystole). However, target temperature may matter in patients with borderline values to minimize post-cardiac arrest syndrome.

Altogether, the results support the clinical relevance of the predictive model and risk score to assist physicians and patients' relatives who deal with difficult decisions after out-of-hospital cardiac arrest due to VF.

\section{Limitations.}

This is a single center study with a limited number of patients, in which survival rates may be higher than expected. However, our data is consistent with similar series in dedicated units that included patients after admission to the ACCU, with an initial rhythm reported as VF and using mild hypothermia.[26, 27] Therefore, the study population may be skewed towards patients with FNP, since patients who died in transit or survived to hospital admission but died in the emergency department or during the hypothermia protocol, before withdrawing sedation, were excluded.

Differences between groups regarding age, gender and family history of sudden cardiac death may have occurred due to the study design. However, the highly reliable predictive performance of the spectral-based model compared with clinical models in the validation cohort, suggests that the model is suitable for clinical practice upon developing appropriate clinical tools. The proposed risk score will nevertheless benefit from further validation in a 
multicenter study with more patients. Additionally, a significant number of traces were not available on admission, which may raise the concern of selection bias. However, our results are consistent with previous studies, which reduces likelihood for such bias.[10, 12, 24, 25] In the future, either incorporating the algorithm into external defibrillators or automated signal transferring from defibrillators to portable medical devices, for signal processing and risk score calculation, may avoid such limitation. The model will also benefit from futures studies aiming at direct comparisons of current biochemical and neurological markers to establish the net benefit on early prognosis.

\section{Conclusions.}

A spectral analysis-based model demonstrates to be highly reliable in predicting in-hospital FNP and survival to discharge in patients with comatose status on admission after cardiac arrest due to VF.

\section{Acknowledgment.}

We thank Dr. Stuart Pocock and Dr. Martín Laclaustra for their assistance with the statistical analysis. 


\section{References.}

[1] Nadkarni VM, Larkin GL, Peberdy MA, Carey SM, Kaye W, Mancini ME, et al. First documented rhythm and clinical outcome from in-hospital cardiac arrest among children and adults. JAMA. 2006;295:50-7.

[2] Cobb LA, Fahrenbruch CE, Olsufka M, Copass MK. Changing incidence of out-ofhospital ventricular fibrillation, 1980-2000. JAMA. 2002;288:3008-13.

[3] Sugawara T, Chan PH. Reactive oxygen radicals and pathogenesis of neuronal death after cerebral ischemia. Antioxid Redox Signal. 2003;5:597-607.

[4] Bernard SA, Gray TW, Buist MD, Jones BM, Silvester W, Gutteridge G, et al. Treatment of comatose survivors of out-of-hospital cardiac arrest with induced hypothermia. N Engl J Med. 2002;346:557-63.

[5] Mild therapeutic hypothermia to improve the neurologic outcome after cardiac arrest. N Engl J Med. 2002;346:549-56.

[6] Lopez-de-Sa E, Rey JR, Armada E, Salinas P, Viana-Tejedor A, Espinosa-Garcia S, et al. Hypothermia in comatose survivors from out-of-hospital cardiac arrest: pilot trial comparing 2 levels of target temperature. Circulation. 2012;126:2826-33.

[7] Rogove HJ, Safar P, Sutton-Tyrrell K, Abramson NS. Old age does not negate good cerebral outcome after cardiopulmonary resuscitation: analyses from the brain resuscitation clinical trials. The Brain Resuscitation Clinical Trial I and II Study Groups. Crit Care Med. 1995;23:18-25.

[8] Bayes de Luna A, Coumel P, Leclercq JF. Ambulatory sudden cardiac death: mechanisms of production of fatal arrhythmia on the basis of data from 157 cases. Am Heart J. 1989;117:151-9. 
[9] Brown CG, Dzwonczyk R, Werman HA, Hamlin RL. Estimating the duration of ventricular fibrillation. Ann Emerg Med. 1989;18:1181-5.

[10] Stewart AJ, Allen JD, Adgey AA. Frequency analysis of ventricular fibrillation and resuscitation success. Q J Med. 1992;85:761-9.

[11] Strohmenger HU, Lindner KH, Lurie KG, Welz A, Georgieff M. Frequency of ventricular fibrillation as a predictor of defibrillation success during cardiac surgery. Anesthesia and analgesia. 1994;79:434-8.

[12] Goto Y, Suzuki I, Inaba H. Frequency of ventricular fibrillation as predictor of oneyear survival from out-of-hospital cardiac arrests. Am J Cardiol. 2003;92:457-9.

[13] Jennett B, Bond M. Assessment of outcome after severe brain damage. Lancet. $1975 ; 1: 480-4$.

[14] Mungas D. In-office mental status testing: a practical guide. Geriatrics. $1991 ; 46: 54-8,63,6$.

[15] Pfeifer R, Borner A, Krack A, Sigusch HH, Surber R, Figulla HR. Outcome after cardiac arrest: predictive values and limitations of the neuroproteins neuron-specific enolase and protein S-100 and the Glasgow Coma Scale. Resuscitation. 2005;65:49-55. [16] A randomized clinical study of cardiopulmonary-cerebral resuscitation: design, methods, and patient characteristics. Brain Resuscitation Clinical Trial I Study Group. Am J Emerg Med. 1986;4:72-86.

[17] Sandroni C, Nolan J, Cavallaro F, Antonelli M. In-hospital cardiac arrest: incidence, prognosis and possible measures to improve survival. Intensive Care Med. $2007 ; 33: 237-45$.

[18] Gallagher EJ, Lombardi G, Gennis P. Effectiveness of bystander cardiopulmonary resuscitation and survival following out-of-hospital cardiac arrest. JAMA. 1995;274:1922-5. 
[19] Wik L, Kramer-Johansen J, Myklebust H, Sorebo H, Svensson L, Fellows B, et al. Quality of cardiopulmonary resuscitation during out-of-hospital cardiac arrest. JAMA 2005;293:299-304.

[20] Martin DR, Brown CG, Dzwonczyk R. Frequency analysis of the human and swine electrocardiogram during ventricular fibrillation. Resuscitation. 1991;22:85-91.

[21] Umapathy K, Foomany FH, Dorian P, Farid T, Sivagangabalan G, Nair K, et al. Realtime electrogram analysis for monitoring coronary blood flow during human ventricular fibrillation: implications for CPR. Heart Rhythm. 2011;8:740-9.

[22] Achleitner U, Wenzel V, Strohmenger HU, Lindner KH, Baubin MA, Krismer AC, et al. The beneficial effect of basic life support on ventricular fibrillation mean frequency and coronary perfusion pressure. Resuscitation. 2001;51:151-8.

[23] Strohmenger HU, Lindner KH, Keller A, Lindner IM, Pfenninger EG. Spectral analysis of ventricular fibrillation and closed-chest cardiopulmonary resuscitation. Resuscitation. 1996;33:155-61.

[24] Freese JP, Jorgenson DB, Liu PY, Innes J, Matallana L, Nammi K, et al. Waveform analysis-guided treatment versus a standard shock-first protocol for the treatment of out-of-hospital cardiac arrest presenting in ventricular fibrillation: results of an international randomized, controlled trial. Circulation. 2013;128:995-1002.

[25] Schoene P, Coult J, Murphy L, Fahrenbruch C, Blackwood J, Kudenchuk P, et al. Course of quantitative ventricular fibrillation waveform measure and outcome following out-of-hospital cardiac arrest. Heart Rhythm. 2014;11:230-6.

[26] Nielsen N, Wetterslev J, Cronberg T, Erlinge D, Gasche Y, Hassager C, et al. Targeted temperature management at 33 degrees $\mathrm{C}$ versus 36 degrees $\mathrm{C}$ after cardiac arrest. N Engl J Med. 2013;369:2197-206. 
[27] Boyce LW, Vliet Vlieland TP, Bosch J, Wolterbeek R, Volker G, van Exel HJ, et al. High survival rate of $43 \%$ in out-of-hospital cardiac arrest patients in an optimised chain of survival. Neth Heart J. 2014. 
Table 1. Baseline Clinical Characteristics

\begin{tabular}{|c|c|c|c|c|}
\hline Clinical Characteristics & $\begin{array}{c}\text { Retrospective } \\
(n=32)\end{array}$ & $\begin{array}{c}\text { Prospective } \\
(n=29)\end{array}$ & $\begin{array}{l}\begin{array}{l}\text { Overall } \\
(n=61)\end{array} \\
\end{array}$ & $\mathbf{P}$ \\
\hline$\overline{\text { Age(years) }}$ & $63.5 \pm 4.5$ & $55 \pm 5.7$ & $55 \pm 3.72$ & 0.0409 \\
\hline Male,n(\%) & $31(96.9)$ & $23(79.4)$ & $54(88.5)$ & 0.031 \\
\hline Family History of SCD, n(\%) & $1(3.57)$ & $8(40.0)$ & $9(15.8)$ & 0.009 \\
\hline Hypertension, $\mathrm{n}(\%)$ & $13(40.6)$ & $15(51.7)$ & $28(45.9)$ & 0.385 \\
\hline Dyslipedemia, n(\%) & $9(28.1)$ & $13(44.8)$ & $22(36.1)$ & 0.174 \\
\hline Diabetes, n(\%) & 7 (21.9) & $3(10.3)$ & $10(16.4)$ & 0.224 \\
\hline Smoking Habit, $n(\%)$ & $12(37.5)$ & $13(44.8)$ & $22(36.1)$ & 0.561 \\
\hline Atrial Fibrillation, $n(\%)$ & $5(15.6)$ & $6(20.7)$ & $11(18)$ & 0.849 \\
\hline Heart Failure, $n(\%)$ & $10(31.3)$ & $6(20.7)$ & $12(26.2)$ & 0.234 \\
\hline Previous Myocardial Infarction, $\mathrm{n}(\%)$ & $6(18.8)$ & $8(27.6)$ & $14(22.9)$ & 0.654 \\
\hline Previous Revascularization, $\mathrm{n}(\%)$ & $4(12.5)$ & $4(13.8)$ & $6(13.1)$ & 0.402 \\
\hline Previous Stroke, $\mathrm{n}(\%)$ & $1(3.1)$ & $1(3.4)$ & $2(3.3)$ & 0.943 \\
\hline Chronic Renal Failure, n(\%) & $2(6.3)$ & $0(0)$ & $2(3.3)$ & 0.271 \\
\hline DCM, n(\%) & $2(6.3)$ & $0(0)$ & $2(3.3)$ & 0.17 \\
\hline COPD, $n(\%)$ & $3(9.4)$ & $5(17.2)$ & $8(13.1)$ & 0.363 \\
\hline $\mathrm{HCM}, \mathrm{n}(\%)$ & $0(0)$ & $3(10.3)$ & $3(4.9)$ & 0.101 \\
\hline Severe Valvulopathy, $n(\%)$ & $2(6.3)$ & $1(3.4)$ & $3(4.9)$ & 0.613 \\
\hline Preexcitation, $\mathrm{n}(\%)$ & $0(0)$ & $1(3.4)$ & $1(1.6)$ & 0.289 \\
\hline Time to ALS, (min) & $8 \pm 2.4$ & $8 \pm 2.0$ & $8 \pm 1.5$ & 0.887 \\
\hline Time performing ALS, (min) & $15 \pm 5.9$ & $10 \pm 5.3$ & $15 \pm 3.9$ & 0.282 \\
\hline Number of shocks delivered before ROSC & $3.5 \pm 1.6$ & $3 \pm 0.87$ & $3 \pm 0.9$ & 0.185 \\
\hline \multicolumn{5}{|l|}{ Background Treatment } \\
\hline Aspirin, $\mathrm{n}(\%)$ & $4(12.5)$ & $5(17.2)$ & $9(14.7)$ & 0.602 \\
\hline Thienopyridines, n(\%) & $1(3.1)$ & $0(0)$ & $1(1.6)$ & 0.524 \\
\hline Betablockers, $\mathrm{n}(\%)$ & $7(21.9)$ & $9(31)$ & $16(26.2)$ & 0.416 \\
\hline Amiodarone, $\mathrm{n}(\%)$ & $2(6.3)$ & $0(0)$ & $2(3.3)$ & 0.271 \\
\hline ACE Inhibitors, $\mathrm{n}(\%)$ & $6(18.8)$ & $7(24.1)$ & $13(21.3)$ & 0.557 \\
\hline ARBs, $n(\%)$ & $1(3.1)$ & $3(10.3)$ & $4(6.6)$ & 0.239 \\
\hline Statins, $\mathrm{n}(\%)$ & $8(25)$ & $10(34.5)$ & $18(29.5)$ & 0.417 \\
\hline Calcium Antagonists, $\mathrm{n}(\%)$ & $1(3.1)$ & $2(6.9)$ & $3(4.9)$ & 0.496 \\
\hline Diuretics, n(\%) & $8(25)$ & $6(20.7)$ & $14(22.9)$ & 0.689 \\
\hline Aldosterone Inhibitors, $\mathrm{n}(\%)$ & $1(3.1)$ & $2(6.9)$ & $3(4.9)$ & 0.476 \\
\hline Anticoagulants, $\mathrm{n}(\%)$ & $5(15.6)$ & $6(20.7)$ & $12(19.7)$ & 0.849 \\
\hline
\end{tabular}

$A C E=$ angiotensin-converting enzyme. $A L S=$ advanced life support. ARBs= Angiotensin-receptor blockers. $\mathrm{COPD}=$ chronic obstructive pulmonary disease. $\mathrm{DCM}=$ Dilated cardiomyopathy. $\mathrm{HCM}=$ Hypertrophic cardiomyopathy. ROSC $=$ Return of spontaneous circulation. 
Table 2. Primary and Secondary Outcomes

\begin{tabular}{|c|c|c|c|c|c|}
\hline Outcome & & $\begin{array}{l}\text { Retrospective } \\
(n=32)\end{array}$ & $\begin{array}{l}\text { Prospective } \\
(n=29)\end{array}$ & $\begin{array}{l}\text { Overall } \\
(n=61)\end{array}$ & $\mathrm{P}\left(\mathrm{X}^{2}\right.$ Test $)$ \\
\hline \multicolumn{6}{|c|}{ Favorable Neurological Performance } \\
\hline & In-Hospital & $16(50.00 \%)$ & $21(72.41 \%)$ & $37(60.66 \%)$ & 0.074 \\
\hline & Follow-Up* & $15(46.88 \%)$ & $19(65.52 \%)$ & $34(55.74 \%)$ & \\
\hline \multicolumn{6}{|l|}{ Survival } \\
\hline & Hospital discharge & $17(53.12 \%)$ & $21(72.41 \%)$ & $38(62.30 \%)$ & 0.121 \\
\hline & Follow-Up* & $16(50.00 \%)$ & $20(68.97 \%)$ & $36(59.02 \%)$ & \\
\hline
\end{tabular}

*Median \pm SEM (25th, 75th percenttiles). Retrospective: $48.5 \pm 10.5(27.0,68.7)$ months. Prospective: $5 \pm 1.8$ (3.5,7.5) months. 
Table 3. Univariate analysis and independent predictive accuracy.

\begin{tabular}{|c|c|c|c|c|}
\hline \multirow[b]{2}{*}{ Categories } & \multirow{2}{*}{$\begin{array}{c}\text { Neurological } \\
\text { Performance } \\
\text { P Value }\end{array}$} & \multicolumn{3}{|c|}{ Univariate Unadjusted Odds Ratio } \\
\hline & & OR (Cl95\%) & Univariate P & $\mathrm{ACC}(\mathrm{Cl} 95)^{*}$ \\
\hline \multicolumn{5}{|l|}{ Clinical Variables } \\
\hline Age & 0.11 & & & \\
\hline Gender & 0.309 & & & \\
\hline Hypertension & 0.281 & & & \\
\hline Dyslipedemia & 0.694 & & & \\
\hline Diabetes & 0.199 & & & \\
\hline Smoking Habit & 0.465 & & & \\
\hline Atrial Fibrillation & 0.07 & $2.158(0.864-5.391)$ & 0.099 & $0.672(0.506-0.838)$ \\
\hline Heart Failure & 0.063 & $1.940(0.945-3.986)$ & 0.071 & $0.655(0.486-0.824)$ \\
\hline Previous Myocardial Infarction & 0.233 & & & \\
\hline Previous Revascularization & 0.219 & & & \\
\hline Previous Stroke & 0.309 & & & \\
\hline Chronic Renal Failure & 0.516 & & & \\
\hline DCM & 0.144 & & & \\
\hline COPD & 0.544 & & & \\
\hline $\mathrm{HCM}$ & 0.565 & & & \\
\hline Severe Valvulopathy & 0.516 & & & \\
\hline $\begin{array}{r}\text { Number of Shocks Delivered before } \\
\text { ROSC }\end{array}$ & 0.023 & $3.025(0.991-9.229)$ & 0.052 & $0.671(0.503-0.837)$ \\
\hline Time to ALS & $<0.001$ & $10.312(2.044-52.032)$ & 0.005 & $0.689(0.524-0.851)$ \\
\hline Time Performing ALS & 0.007 & $2.770(1.046-7.336)$ & 0.04 & $0.676(0.509-0.84)$ \\
\hline \multicolumn{5}{|l|}{ Background Treatment } \\
\hline Aspirin & 0.285 & & & \\
\hline Thienopyridines & 0.309 & & & \\
\hline Betablockers & 0.669 & & & \\
\hline Amiodarones & 0.144 & & & \\
\hline ACE Inhibitors & 0.365 & & & \\
\hline ARBs & 0.309 & & & \\
\hline Statins & 0.414 & & & \\
\hline Calcium Antagonists & 0.31 & & & \\
\hline Diuretics & 0.314 & & & \\
\hline Anticoagulants & 0.133 & & & \\
\hline Aldosterone Inhibitors & 0.309 & & & \\
\hline \multicolumn{5}{|l|}{ VF Variables } \\
\hline \multicolumn{5}{|l|}{ Fundamental Spectral Variables } \\
\hline Dominant Frequency & $<0.001$ & $0.089(0.020-0.403)$ & 0.002 & $0.884(0.77-0.988)$ \\
\hline Median Frequency & $<0.001$ & $0.073(0.015-0.355)$ & 0.001 & $0.86(0.741-0.98)$ \\
\hline Normalized $80 \%$ PSD & 0.005 & $3.121(1.270-7.669)$ & 0.013 & $0.579(0.4-0.75)$ \\
\hline 1Hz DF Spectral Concentration & 0.023 & $2.198(1.027-4.704)$ & 0.042 & $0.634(0.463-0.805)$ \\
\hline Amplitude Spectrum Area (AMSA) & $<0.001$ & $0.053(0.008-0.362)$ & 0.003 & $0.85(0.723-0.977)$ \\
\hline Spectral Regularity Index & 0.07 & & & \\
\hline Derived Spectral Variables & & 2 & & \\
\hline High-to-Low Peak Ratio & $<0.001$ & $0.073(0.014-0.372)$ & 0.002 & $0.817(0.678-0.954)$ \\
\hline High-to-Low PSD Ratio & $<0.001$ & $0.034(0.003-0.396)$ & 0.007 & $0.847(0.721-0.969)$ \\
\hline \multicolumn{5}{|l|}{ Time Domain } \\
\hline Mean Amplitude & 0.11 & & & \\
\hline $\begin{array}{r}\text { Approximate Entropy Regularity } \\
\text { Index }\end{array}$ & $<0.001$ & $0.032(0.003-0.355)$ & 0.005 & $0.796(0.652-0.937)$ \\
\hline
\end{tabular}

* Univariate predictive accuracy averaged for training (Group 1, retrospective) and validation (Group 2, prospective). OR represent univariate odds ratios for non-FNP. AMSA: Amplitude spectrum area. PSD: Power spectral density. ROSC: Return of spontaneous circulation. Other abbreviations as in Table 1. 
Table 4. Multivariate best performance model.

\begin{tabular}{lcc}
\hline Variable & $\begin{array}{c}\text { Multivariate adjusted Odds } \\
\text { Ratio for non-FNP (OR) }\end{array}$ & Cl 95\% \\
\hline Dominant Frequency (Hz) & 0.252 & $0.227-0.281$ \\
High-to-Low Peak Ratio (A.U.) & 0.164 & $0.145-0.185$ \\
High-to-Low PSD Ratio (A.U.) & 0.264 & $0.237-0.295$ \\
Number of shocks delivered & 5.14 & $4.79-5.51$ \\
before ROSC & &
\end{tabular}

Abbreviations as in Table 3. 
Table 5. In-hospital performance of the best multivariate predictive model.

Se Sp C-Stat ACC

\section{Favorable Neurological Performance}

$\begin{array}{lllll}\text { Retrospective group (Training) } & 0.94 & 1 & 0.98 & 0.97 \\ \text { Prospective group (Validation) } & 0.88 & 0.91 & 0.89 & 0.90\end{array}$

Survival Outcome (Test)

Retrospective group

$0.94 \quad 0.94 \quad 0.95 \quad 0.93$

Prospective group

\begin{tabular}{llll}
0.88 & 0.91 & 0.92 & 0.89 \\
\hline
\end{tabular}




\section{Figure legends.}

Figure 1. Digitization and signal processing of a representative VF trace. A. Upper panel, single lead VF trace from paper ECG prior to the first DC shock. Lower panel, 5-s VF epoch after digitization, segmentation and codification. B. Representative spectra of the VF trace showed in A. DF, MF, 1-Hz DF spectral concentration and PSD 80\%, are shown. The univariate cut-off at $3.9 \mathrm{~Hz}$ was used to define low and high PSD bands. DF= dominant frequency. $\mathrm{MF}=$ median frequency. $\mathrm{NSC}=$ normalized spectral concentration. $\mathrm{PSD}=$ power spectral density.

Figure 2. Workflow of patients included in group 1 and group 2.

Figure 3. A. Power spectral density (PSD) of all patients with in-hospital favorable (A1) and non-favorable (A2) neurological performance. The patients are sorted based on their DF values. DF peaks are pointed out for each individual (black vertical dashed-line). The cut-off threshold of $3.9 \mathrm{~Hz}$ was chosen for color-coding above (red) and below (blue) the PSD. B. Boxplot representation of DF comparing patients from both groups for primary and secondary endpoints. Boxes depict median and interquartile range (25-75\%). Red dots are outliers at least twice the interquartile range from the median. Green dots represent outliers to hospital discharge who improved neurological performance during follow-up.

Figure 4. Risk score based on the predictive performance of the model. A. Observed (triangles) and predicted (circles) probability of FNP for the entire population. Blue and red represent FNP and non-FNP, respectively (dark fill, retrospective; light fill, prospective). We defined four risk groups of non-FNP performance according to their risk scores as follows: expected FNP; very low (VL) and low risk (L) and expected non-FNP; high (H) and very high risk (VH). B. Percentage of patients (observed, dark gray and predicted, light gray) who belong to each of the risk score groups in both the retrospective (B1) and 
prospective cohorts (B2). $(\alpha)$ and $(\beta)$ represent false negative and false positive individuals, respectively. $\mathrm{FNP}=$ favorable neurological performance. 


\section{Supplemental Material.}

\section{Spectral Analysis-Based Risk Score Enables Early Prediction of Mortality and Cerebral Performance in Patients Undergoing Therapeutic Hypothermia for Ventricular Fibrillation and Comatose Status.}

David Filgueiras-Rama*,a,c,d,1,2 , Conrado J. Calvo ${ }^{\mathrm{b}, \mathrm{e}, 2,3}$, Óscar Salvador-Montañés ${ }^{\mathrm{d}, 3,4}$, Rosalía Cádenas $^{\mathrm{d}, 4}$, Jose Ruiz-Cantador ${ }^{\mathrm{d}, 4}$, Eduardo Armada ${ }^{\mathrm{d}, 4}$, Juan Ramón Rey ${ }^{\mathrm{d}, 4}$, JL Merino ${ }^{\mathrm{d}, 4}$, Rafael Peinado $^{\mathrm{d}, 4}$, Nicasio Pérez-Castellano ${ }^{\mathrm{c}, 3}$, Julián Pérez-Villacastín ${ }^{\mathrm{c}, 3}$, Jorge G. Quintanilla ${ }^{\mathrm{a}, 3}$, Santiago Jiménez, ${ }^{\mathrm{b}, 3}$, Francisco Castells $\mathrm{s}^{\mathrm{b}, \mathrm{e}, 3}$, Francisco J. Chorro ${ }^{\mathrm{e}, 3}$, JL López-Sendón ${ }^{\mathrm{d}, 3}$, Omer

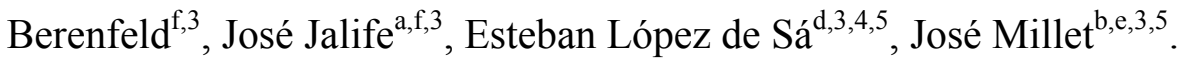

${ }^{a}$ Atherothrombosis, Imaging and Epidemiology Department. Fundación Centro Nacional de Investigaciones Cardiovasculares (CNIC), Madrid, Spain.

b Bio-ITACA Grupo de Bioingeniería, Instituto de Aplicaciones Avanzadas, Dpto Ingeniería Electrónica, Universitat Politècnica de Valencia, Spain

${ }^{\mathbf{c}}$ Hospital Clínico San Carlos, Madrid, Spain.

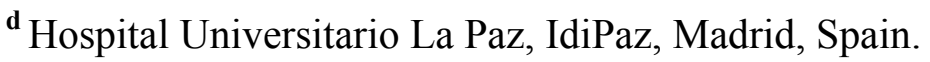

${ }^{\mathbf{e}}$ Grupo Electrofisiología y Bioingeniería, Microcluster en Protección Cardiovascular, Dpto de Medicina, Fundación INCLIVA Hospital Clínico, Valencia, Spain

${ }^{\text {f }}$ Center for Arrhythmia Research. Cardiovascular Research Center, Department of Internal Medicine, University of Michigan, Ann Arbor, USA. 


\section{Supplemental Methods.}

\section{Analysis of ventricular fibrillation traces.}

All data analyses, extraction and quantifications were done using custom scripts written in MATLAB software (version 7.11.0 R2010b, The MathWorks Inc, Natick, Massachusetts, USA). The scripts were developed using the retrospective cohort. Blinded investigators to clinical outcomes performed waveform and spectral analysis of ventricular fibrillation (VF) traces.

\section{a) Image digitization, signal extraction and interpretation.}

Standard ECG tape recordings were used to assess waveform parameters of VF prior to the first documented DC shock in all patients. Lead II traces were extracted unless artifacts were present. A semi-automatic approach was used for digitization using a Matlab-based custom tool. VF recordings were scanned to a digital image (1200 dpi) and stored in a codified digital format. To identify VF traces on the standard ECG-paper, image-processing techniques were applied involving binary thresholding, pixel-to-point conversion and transform techniques.[1,2] When necessary, images were pre-processed to avoid artifacts (contrast, median filtering and interpolation) in the digitization process. Pixel-to-point conversion was applied using the lower waveform envelope. The graphical grid was used as reference for interpretation. Before signal processing, an independent investigator reviewed all digitized traces to ensure accurate extraction of VF deflections.

\section{b) Signal processing.}

Digitized signals were post-processed and further analyzed in Matlab. Up to 5-s artifact-free segments prior to the external DC shock were extracted in each patient. Signals with less duration were zero-padded up to $5 \mathrm{~s}$. Only segments of at least $3 \mathrm{~s}$ were eligible for the study (Mean $\pm \operatorname{Std}$ duration $3.91 \pm 0.98 \mathrm{~s}, \mathrm{~N}=61$ ). All signals were detrended by mean removal. A $4^{\text {th }}$ order polynomial approximation was applied to remove the baseline trend when appropriate. Then, VF 
tracings were bandpass filtered between 1.5 and $40 \mathrm{~Hz}$ using $10^{\text {th }}$ order Butterworth digital filters. Averaged power spectral density (PSD) was obtained at each frequency. We used a nonparametric Welch method for robust Fast Fourier Transform (FFT) estimation with $0.2 \mathrm{~Hz}$ spectral resolutions in the 1.5 to $10 \mathrm{~Hz}$ band of interest, where at least $90 \%$ of the spectral power was concentrated. Spectral information in this band was normalized to the total power for each patient before attempting to extract any spectral variable.

\section{c) Extracting temporal and spectral variables.}

Both time and frequency domain pre-selected variables were extracted and quantified across patients. Fundamental spectral variables for VF were measured and calculated as described elsewhere.[3-9] Dominant frequency (DF) was defined as the frequency with the highest power in the 1.5 to $10 \mathrm{~Hz}$ band of interest. Median frequency (MF) describes the power distribution in the frequency spectrum and it was calculated as the frequency cut-off where $50 \%$ of the power in the spectrum was below (and above) in the $1.5-10 \mathrm{~Hz}$ band. Normalized spectral concentration at DF, was obtained both at $80 \%$ of the maximum PSD ( $\left.\mathrm{PSD}_{80 \%}\right)$ and through all power density contained in the band $\mathrm{DF} \pm 1 \mathrm{~Hz}$ (NSC).[7] Spectral regularity index (RI) was defined as the ratio between the power at DF and the power in the 1.5 to $10 \mathrm{~Hz}$ band.[8] The Amplitude Spectral Area (AMSA) was calculated as the summed product of contributing frequencies weighted by the absolute value of power at that frequency from the PSD Welch spectrum (1.5 to $10 \mathrm{~Hz}$ band).[10] After univariate binary logistic regression analysis on the retrospective cohort, DF offered the best independent prediction accuracy (proportion of true results, both true positives and true negatives, in the population). A cut-off at $3.9 \mathrm{~Hz}$ showed the highest sensitivity (0.88) and specificity (0.94) for predicting the primary endpoint in the retrospective cohort. Therefore, we used the $3.9 \mathrm{~Hz}$ cut-off to obtain two additional derived ratios of spectral variables as follows:[11] High-to-Low Power Spectral Density Ratio (HL-PSDR) as the relative power 
between high $(3.9$ to $10 \mathrm{~Hz})$ and low $(1.5$ to $3.9 \mathrm{~Hz})$ bands, and High-to-Low Peak Ratio (HL$\mathrm{pKR})$ as the relative the number of spectral peaks above and below the $3.9 \mathrm{~Hz}$ threshold with power above $40 \%$ the frequency with the highest power (DF).

We also considered time-domain waveform variables for the study. We included the mean amplitude over time (A) and the Approximate Entropy Regularity Index (AppEnt), which measures the complexity of a time sequence as the natural logarithm of the relative prevalence of repetitive patterns found within the sequence.[12, 13]

For multivariate analysis, we selected non-correlated statistically significant variables among all extracted VF fundamental variables. Spearman and Pearson correlations were done to determine monotonic and linear relationships in the retrospective cohort $(p<0.001)$. Highly correlated (Rho and $\mathrm{R}>0.9$ ) variables were considered for elimination (Supplemental Figure 1), leaving those with the highest univariate prediction accuracy for the primary outcome.

\section{Statistical analysis and prediction model.}

All values are presented as median $\pm \operatorname{SEM}\left(25^{\text {th }}, 75^{\text {th }}\right.$ percentiles $)$ except where noted. Group 1 (retrospective cohort) was used to develop an algorithm to predict the primary outcome. Each of the clinical, spectral and time-domain VF variables underwent univariate analysis to evaluate its association with in-hospital FNP. Normal distribution of variables was assessed with the Shapiro-Wilk test. Statistical significance was assessed by the parametric T-test or the nonparametric Mann-Whitney-Wilcoxon test, as appropriate. If necessary, we used Bonferroni correction for multiple comparisons. Categorical variables and percentile comparisons were performed using a Chi-squared test or the Fisher Exact test, as appropriate. $\mathrm{P}<0.05$ was considered statistically significant. Non-correlated variables, among statistically significant ones, and clinically-relevant variables were regressed out against the primary outcome by using a 
stepwise backward multivariate logistic regression approach.[14, 15] All variables were standardized before entering into the multivariate analysis. We considered clinically-relevant variables to avoid bias, even though some were non-significant or not associated to the primary outcome. Interestingly, the multivariate analysis resulted in the same prediction model either including all relevant clinical variables or not. Based on the prior correlation results and clinical relevance, all variables considered in the multivariate analyses were the following:

- Clinical variables: Atrial fibrillation, heart failure, number of shocks delivered before return of spontaneous circulation (ROSC), time to advance life support (ALS), time performing ALS, age, previous myocardial infarction (MI), previous stroke, chronic renal failure.

- Fundamental and derived VF variables with the highest univariate prediction accuracy among highly correlated variables: DF, HL-pKR, HL-PSDR, NSC, PSD $80 \%$.

The multivariate logistic regression was performed using a backward iterative approach to eliminate variables and search for the best performance model, which was determined by the ROC curves obtained for each classification (maximum squared root product of sensitivity and specificity and maximum C-stat). We aimed at predicting the primary outcome with the highest sensitivity and specificity achievable using the minimum number of variables that guarantied the best performance and predictive accuracy (ACC, percentage of correctly classified observations). Such a predictive model was validated on the prospective cohort. The model was also tested on both cohorts to predict outcomes at follow-up.

To correct for bias, confidence intervals (95\%) were found using bias corrected and accelerated bootstrap.[16] Robustness of the correlation between the outcome and expected outcome was challenged using the Jackknife approach to ensure stability beyond individual observations showing that the classification was significantly different from a random classification $(\mathrm{p}<0.001)$. 
Finally, patients from both groups were categorized according to their risk scores obtained in the multivariate analysis. Using the best probability threshold obtained by the prediction model on the retrospective cohort we defined four risk subsets of the population for non-FNP as follows: very low and low risk of non-FNP (expected FNP), high and very high risk of non-FNP (expected non-FNP).

In order to assess the relative contribution of the spectral parameters within the best prediction model (Model I) we evaluated a model built from the spectral predictors alone (Model II) and compared its performance and overall accuracy with: a) a model built using the only clinical variable in our prediction model (Model III), b) a model built from the most associated clinical variables to the primary outcome (FNP) in the retrospective cohort (Model IV), c) a model including both associated clinical variables and the most relevant clinical variables (Model V) (Supplemental Table 6). Variables included after backward elimination in Model V are described below.

- Model I (Best performance model, 4 variables): DF, HL-PSDR, HL-pKR and number of shocks delivered before ROSC.

- Model II (3 spectral variables): DF, HL-PSDR and HL-pKR.

- Model III (1 clinical variable): number of shocks delivered before ROSC.

- Model IV (Best clinical model, 5 variables): number of shocks delivered before ROSC, time to ALS, time performing ALS, heart failure (HF), history of atrial fibrillation (AF).

- Model V (Associated and clinical relevant variables, 8 variables): age, number of shocks delivered before ROSC, time performing ALS, HF, history of previous MI, history of AF, history of previous stroke and chronic renal failure. Time to ALS and LVEF after hypothermia were excluded automatically by the backward elimination approach.

All analyses were done using SSPS v21 and custom Matlab scripts for mathematical assistance. 


\section{References}

[1] Ravichandran L, Harless C, Shah AJ, Wick CA, McLellan JH, Tridandapani S. Novel Tool for Complete Digitization of Paper Electrocardiography Data. IEEE Journal of Translational Engineering in Health and Medicine. 2013:1800107-.

[2] Kumar DG, Thakur D, Sharma S, Bhardwaj S. ECG Paper Records Digitalization through Image Processing Techniques. Int J Comput Appl 2012;48:35-8.

[3] Brown CG, Dzwonczyk R. Signal analysis of the human electrocardiogram during ventricular fibrillation: frequency and amplitude parameters as predictors of successful countershock. Ann Emerg Med. 1996;27:184-8.

[4] Strohmenger HU, Eftestol T, Sunde K, Wenzel V, Mair M, Ulmer H, et al. The predictive value of ventricular fibrillation electrocardiogram signal frequency and amplitude variables in patients with out-of-hospital cardiac arrest. Anesth Analg. 2001;93:1428-33.

[5] Eftestol T, Sunde K, Ole Aase S, Husoy JH, Steen PA. Predicting outcome of defibrillation by spectral characterization and nonparametric classification of ventricular fibrillation in patients with out-of-hospital cardiac arrest. Circulation. 2000;102:1523-9.

[6] Reed MJ, Clegg GR, Robertson CE. Analysing the ventricular fibrillation waveform. Resuscitation. 2003;57:11-20.

[7] Such-Miquel L, Chorro FJ, Guerrero J, Trapero I, Brines L, Zarzoso M, et al. Evaluation of the complexity of myocardial activation during ventricular fibrillation. An experimental study. Rev Esp Cardiol. 2013;66:177-84.

[8] Kalifa J, Tanaka K, Zaitsev AV, Warren M, Vaidyanathan R, Auerbach D, et al. Mechanisms of wave fractionation at boundaries of high-frequency excitation in the posterior left atrium of the isolated sheep heart during atrial fibrillation. Circulation. 2006;113:626-33. 
[9] He M, Chen B, Gong Y, Wang K, Li Y. Prediction of Defibrillation Outcome by Ventricular Fibrillation Waveform Analysis. A Clinical Review. J Clinic Experiment Cardiol. 2013.

[10] Schoene P, Coult J, Murphy L, Fahrenbruch C, Blackwood J, Kudenchuk P, et al. Course of quantitative ventricular fibrillation waveform measure and outcome following out-of-hospital cardiac arrest. Heart Rhythm. 2014;11:230-6.

[11] Sherman LD. The frequency ratio: an improved method to estimate ventricular fibrillation duration based on Fourier analysis of the waveform. Resuscitation. 2006;69:479-86.

[12] Weaver WD, Cobb LA, Dennis D, Ray R, Hallstrom AP, Copass MK. Amplitude of ventricular fibrillation waveform and outcome after cardiac arrest. Ann Intern Med. 1985;102:535.

[13] Ho KK, Moody GB, Peng CK, Mietus JE, Larson MG, Levy D, et al. Predicting survival in heart failure case and control subjects by use of fully automated methods for deriving nonlinear and conventional indices of heart rate dynamics. Circulation. 1997;96:842-8.

[14] Cole TJ. Applied logistic regression. In: Hosmer DW, and Lemeshow S, editors. Statist Med. New York: Wiley; 1991. p. 1162-3.

[15] Adrie C, Cariou A, Mourvillier B, Laurent I, Dabbane H, Hantala F, et al. Predicting survival with good neurological recovery at hospital admission after successful resuscitation of out-of-hospital cardiac arrest: the OHCA score. Eur Heart J. 2006;27:2840-5.

[16] DiCiccio TJ, Efron B. Bootstrap confidence intervals. Statist Sci. 1996;11:189-212. 


\section{Supplemental Figure legends.}

Supplemental Figure 1. Correlation analysis of spectral variables. Both linear (R Pearson) and monotonic (Rho Spearman) correlation coefficients were calculated among spectral and temporal ventricular fibrillation variables significantly associated to favorable neurological performance. Arrows highlight strong linear and monotonic correlation between DF and MF, AMSA and AppEnt in the retrospective cohort. AMSA $=$ amplitude spectrum area. AppEnt= Approximate Entropy Regularity Index. DF= dominant frequency. HLpKR= High-to-Low peak ratio. HLPSDR $=$ High-to-Low $\mathrm{PSD}$ ratio. $\mathrm{MF}=$ median frequency. $\mathrm{NSC}=$ normalized spectral concentration. $\mathrm{PSD}=$ power spectral density. $\mathrm{RI}=$ Spectral regularity index.

Supplemental Figure 2. Causes of ventricular fibrillation across population $(\mathrm{N}=61)$.

Supplemental Figure 3. Individual outcomes and follow-up. A. Quadrant-based representation of outcomes and follow-up of each individual patient. Patients are sorted in each quadrant whether they belong to the retrospective (top, R) or prospective (bottom, P) cohorts. Follow-up (F) of patients who survived to hospital discharge $(\mathrm{H})$ is shown on the right for each group. Transitions during follow-up are indicated with a dashed line. Each color represents one patient. Markers represent each quadrant condition (circles; FNP+S, downward triangle; FNP+Non-S, upward triangle; Non-FNP+S and squares; Non-FNP+Non-S). B. Pie charts showing deaths at follow-up and deaths despite FNP. FNP= Favorable neurological performance. S= Survival.

Supplemental Figure 4. ROC curves for the best performance prediction model. A. ROC curve for in-hospital favorable neurological performance in the retrospective cohort $(\mathrm{C}$-stat $=0.98)$. B. ROC curve for the secondary end-point (survival) in the retrospective cohort $(\mathrm{C}$-stat $=0.95)$. C. 
ROC curve for the primary outcome in the prospective cohort. (C-stat $=0.89)$. D. ROC curved for survival to hospital discharge in the prospective cohort $(\mathrm{C}$-stat $=0.92)$.

Supplemental Figure 5. Individual variability of spectral predictors and risk score. A. VF tracing with superimposed 3-s sliding windows that were used to obtain spectral components from the DC shock up to the available signal length (maximum available=12 s). VF spectrum was obtained for each segment and the spectral components were estimated. B. Sequential spectral components along 3-s segments ('t' seconds prior to the DC shock) in a patient with FNP (B1) and a patient with non-FNP (B2). Both patients showed consistent spectral components in time. C. Degree of variability on spectral components assessed by the standard deviation (SD) of the difference on each measure for all segments, over absolute mean of the individual risk score for each patient. Despite the individual variability, all patients were classified within their stratified risk score group. D. Superimposed risk score classification for neurological performance based on the probability assigned by the multivariate model using both the averaged estimation for each predictor and the single up-to-5s segment estimation. Risk score reproducibility was confirmed by comparison of the outcome results between both estimations. Both approaches showed the same derived statistics (sensitivity, specificity, C-stat) and best performance threshold for classification. Confidence interval (IC95) is shown in shaded blue for the averaged risk score fitting. VF= ventricular fibrillation. FNP as in Supplemental Figure 3.

Supplemental Figure 6. DF and risk score of the study population compared with a control population of VF patients without comatose status after DC shock. A. Boxplot quantification of DF in patients with $\mathrm{DF} \geq \mathrm{Th}(\mathrm{n}=39), \mathrm{DF}<\mathrm{Th}(\mathrm{n}=22)$ and Controls $(\mathrm{n}=11)$ (Threshold=3.9 Hz). Individual observations (patients) are shown in blue circles spread over the boxplots. Significant 
differences were found between Controls and patients with $\mathrm{DF}<\mathrm{Th}(\mathrm{p}<0.001)$. B. Risk score classification for all patients from each group. All Controls were properly classified within favorable neurological performance, as expected. Red dots are outliers at least twice the interquartile range $(25-75 \%)$ from the median. $\mathrm{Th}=$ Threshold. $\mathrm{VF}=$ ventricular fibrillation.

Supplemental Figure 7. Comparison of the number of shocks delivered during resuscitation between groups with high and low DF. A. A significantly higher number of shocks delivered before ROSC was present in patients with DF values< $<$ Threshold (Th) $(3.9 \mathrm{~Hz})$. Individual observations (patients) are shown in blue circles spread over the boxplots. Red dots are outliers at least twice the interquartile range (25-75\%) from the median. B. No significant differences between groups were found in the number of shocks delivered before conversion to non-VF rhythms. DF and VF as in Supplemental Figures 1 and 5, respectively.

Supplemental Figure 8: Representative VF recordings with a DF peak close to the cut-off value (3.9 Hz). Upper panel, digitized trace before the first DC shock. Lower panel, representative spectra of the VF signal. Blue color filing shows low spectral bands (1.5 to $3.9 \mathrm{~Hz}$ ) and red filling shows high spectral bands. Despite a DF peak at $3.97 \mathrm{~Hz}$, both HL-PSDR (2.12) and HL-pKR (3) aided in correctly classifying such a case within low risk of non-favorable neurological performance: Risk score Log (odds); Log (-1.79). DF and VF as in Supplemental Figures 1 and 5, respectively. 
Supplemental Figure 1

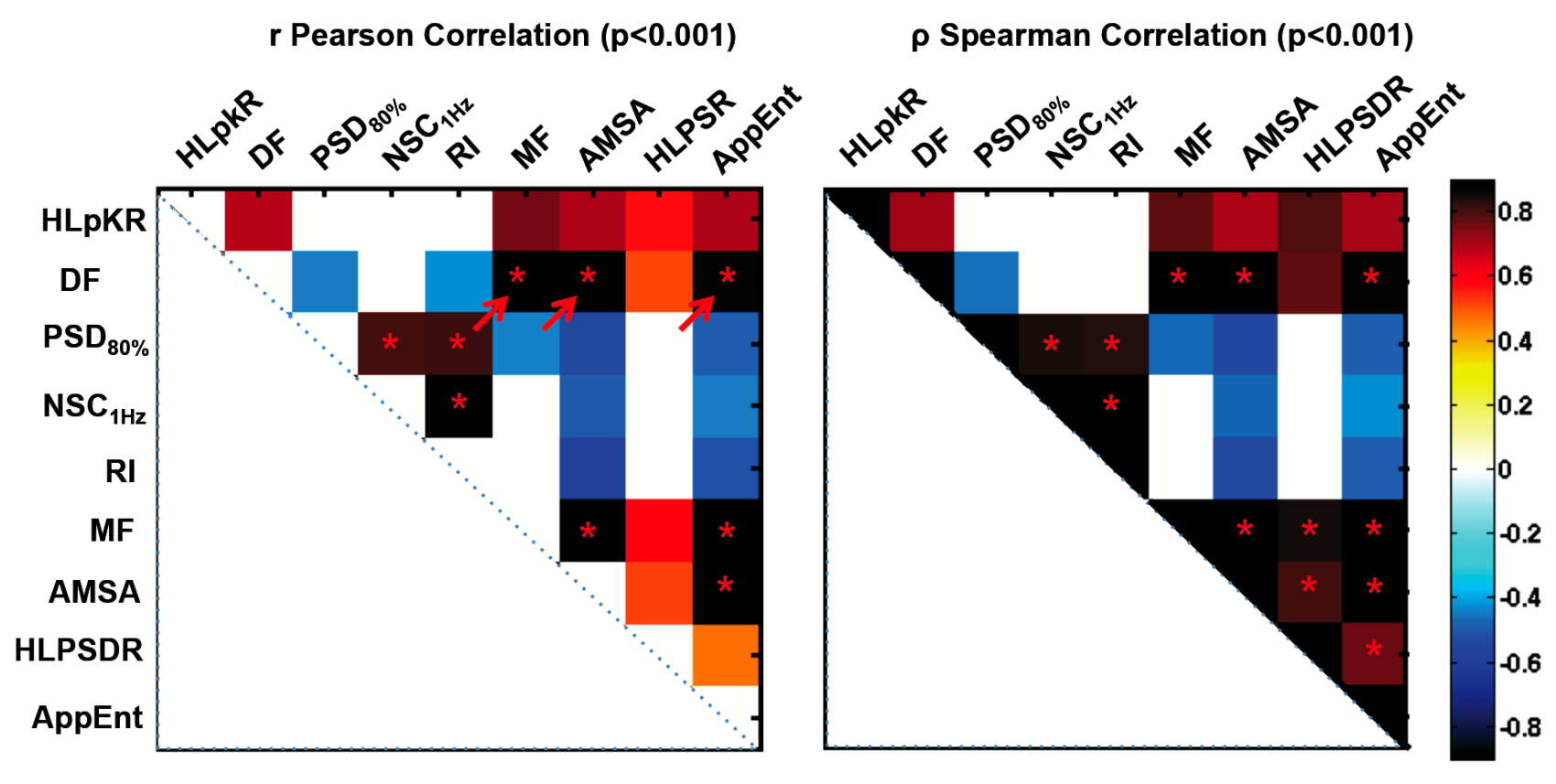




\section{Supplemental Figure 2}

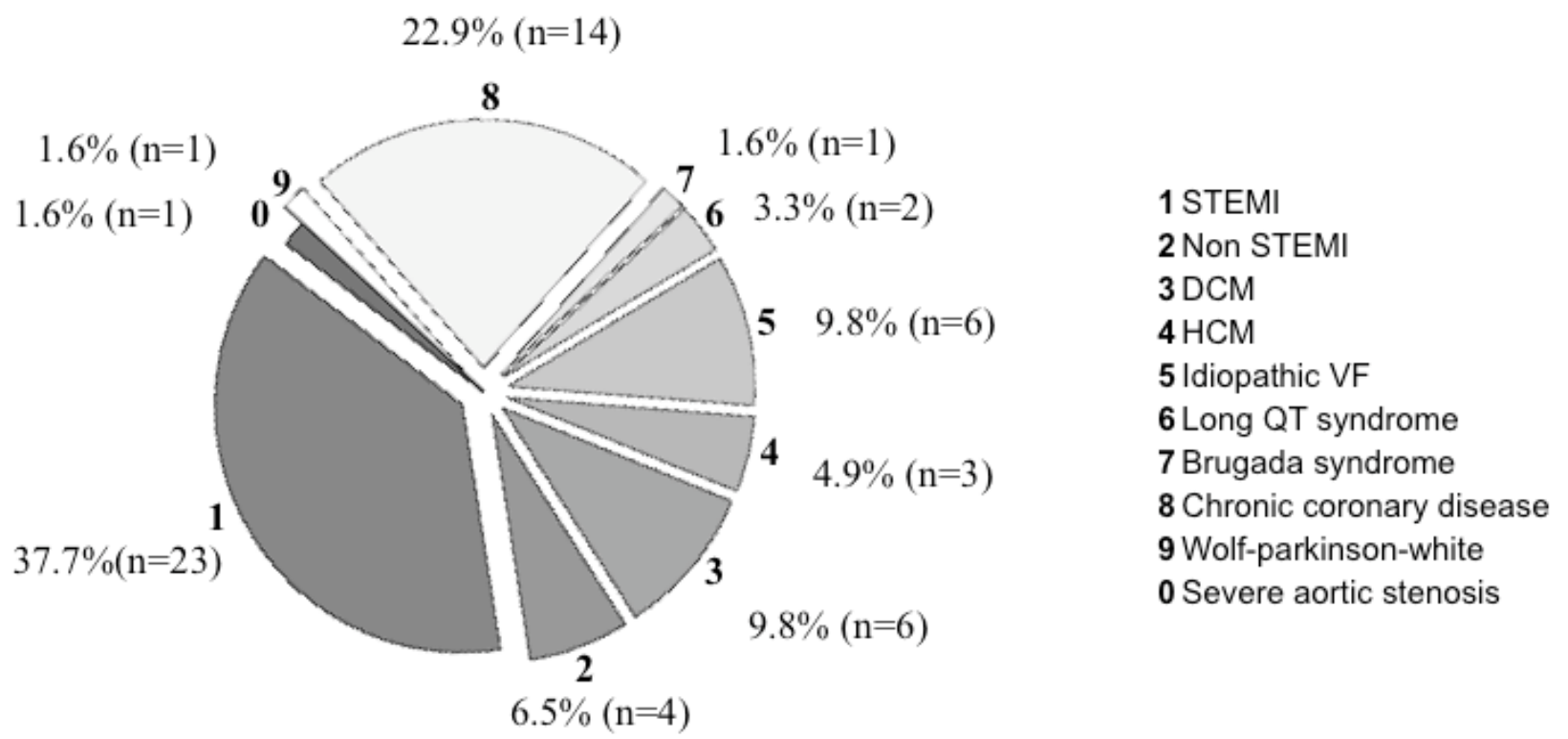


Supplemental Figure 3

A

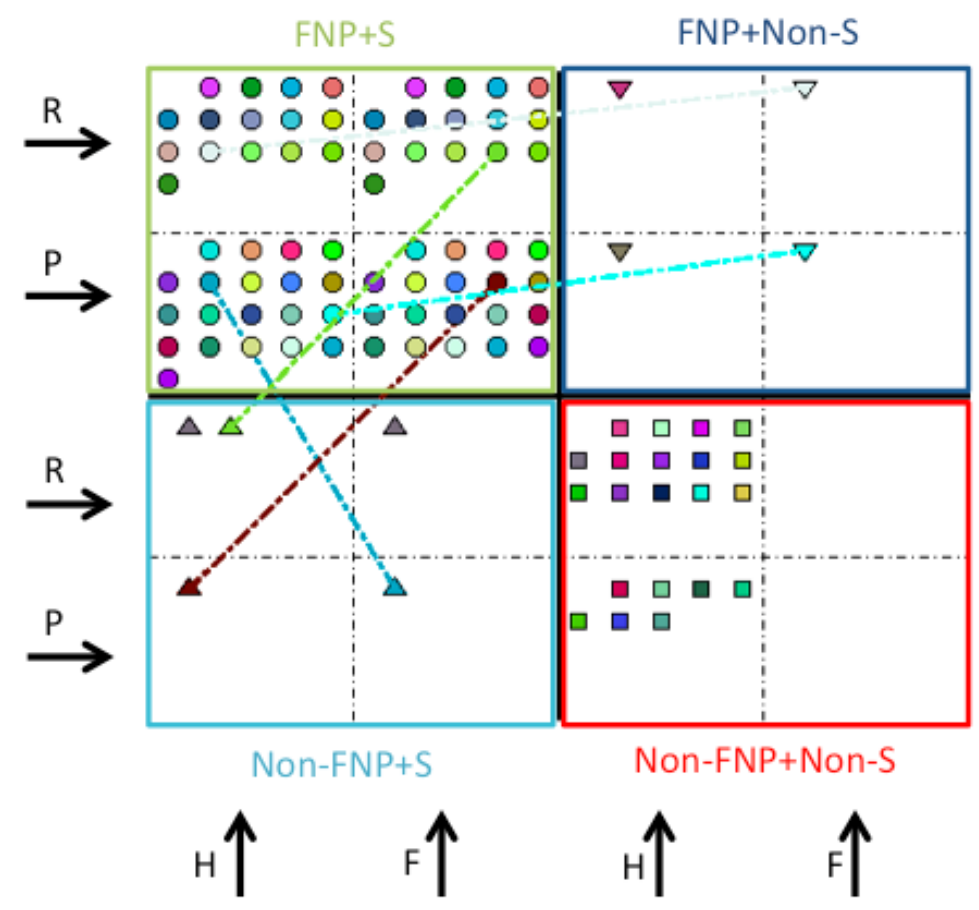

B

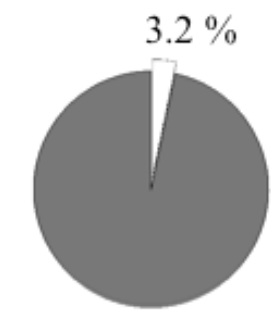

Deaths during follow up

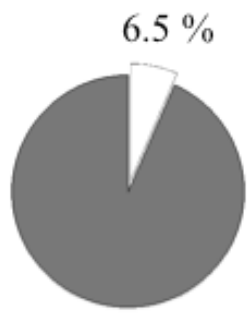

Deaths despite favorable neurological performance 


\section{Supplemental Figure 4}
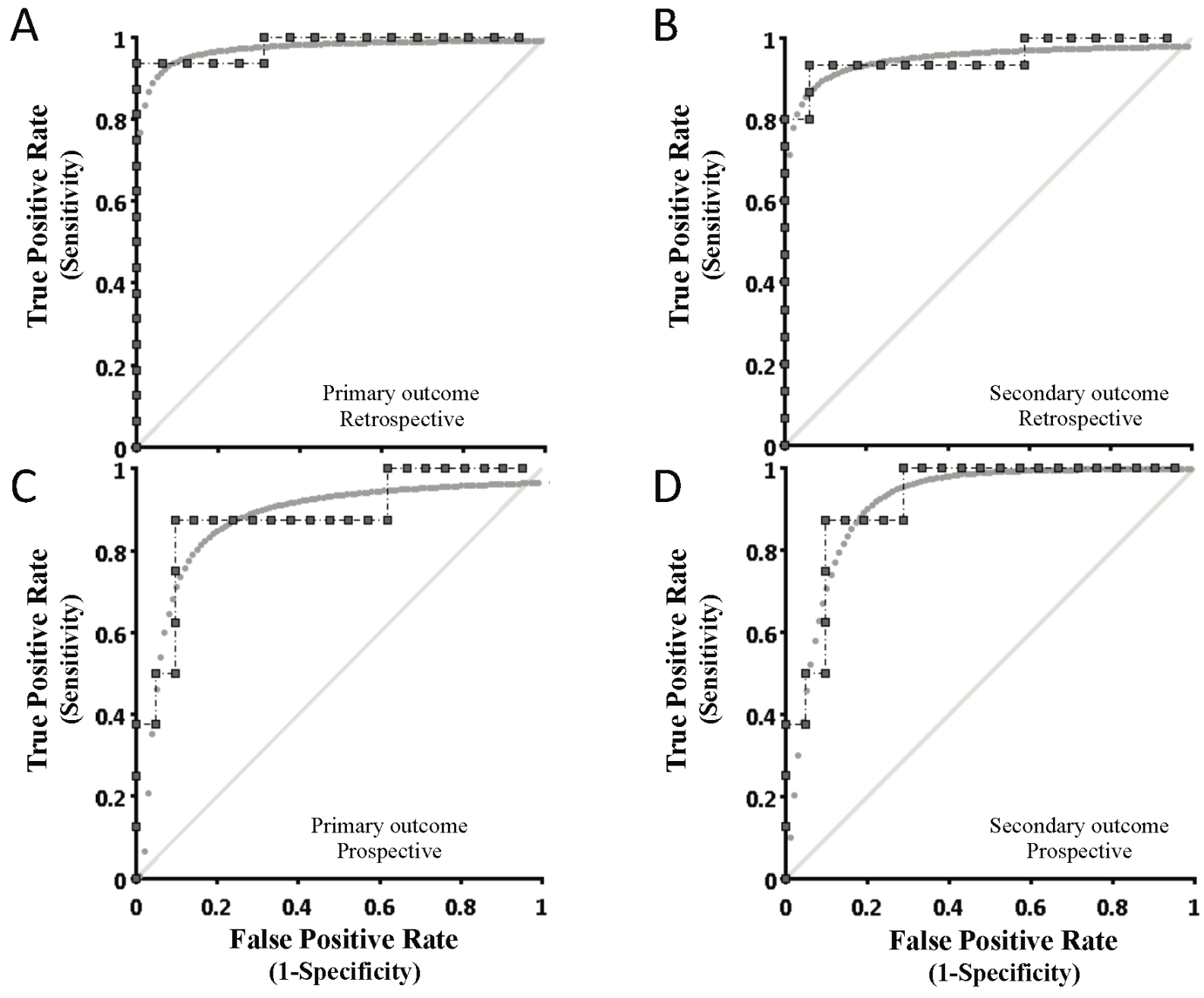


\section{Supplemental Figure 5}

A

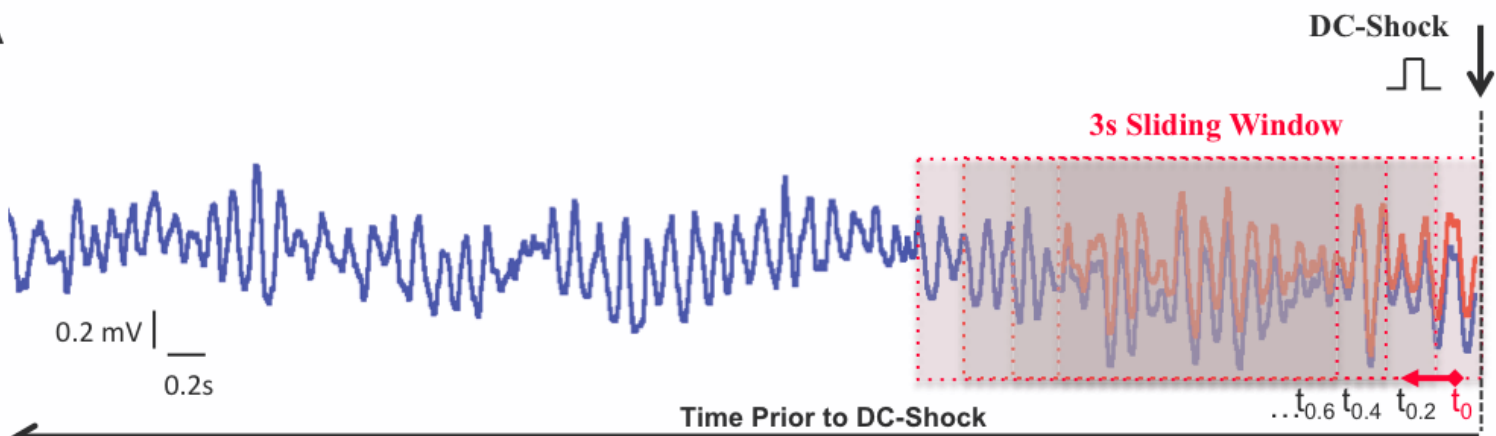

$\mathrm{B}_{\mathrm{B} 1}$

Time Prior to DC-Shoc

Patient with FNP

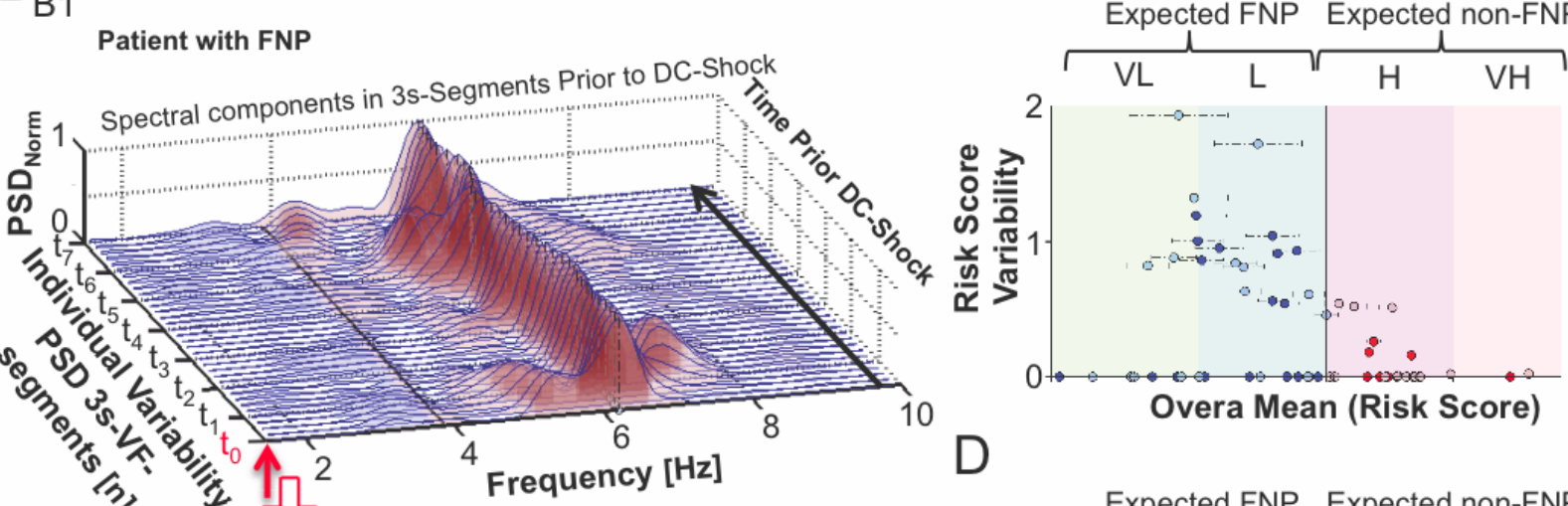

B2

Patient with non-FNP

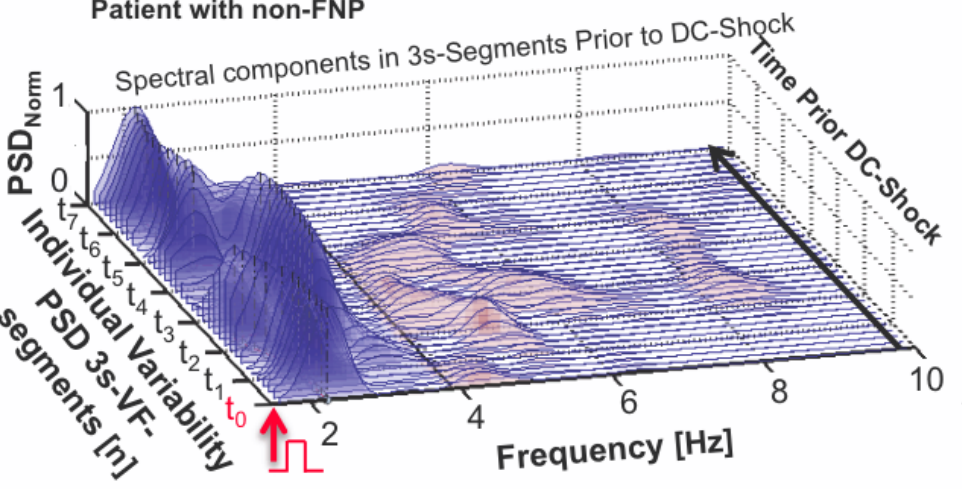

C

D Expected FNP Expected non-FNP

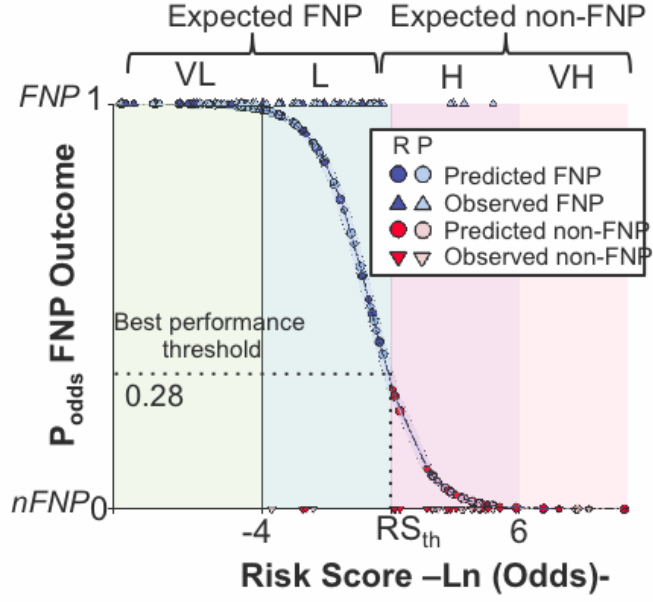




\section{Supplemental Figure 6}
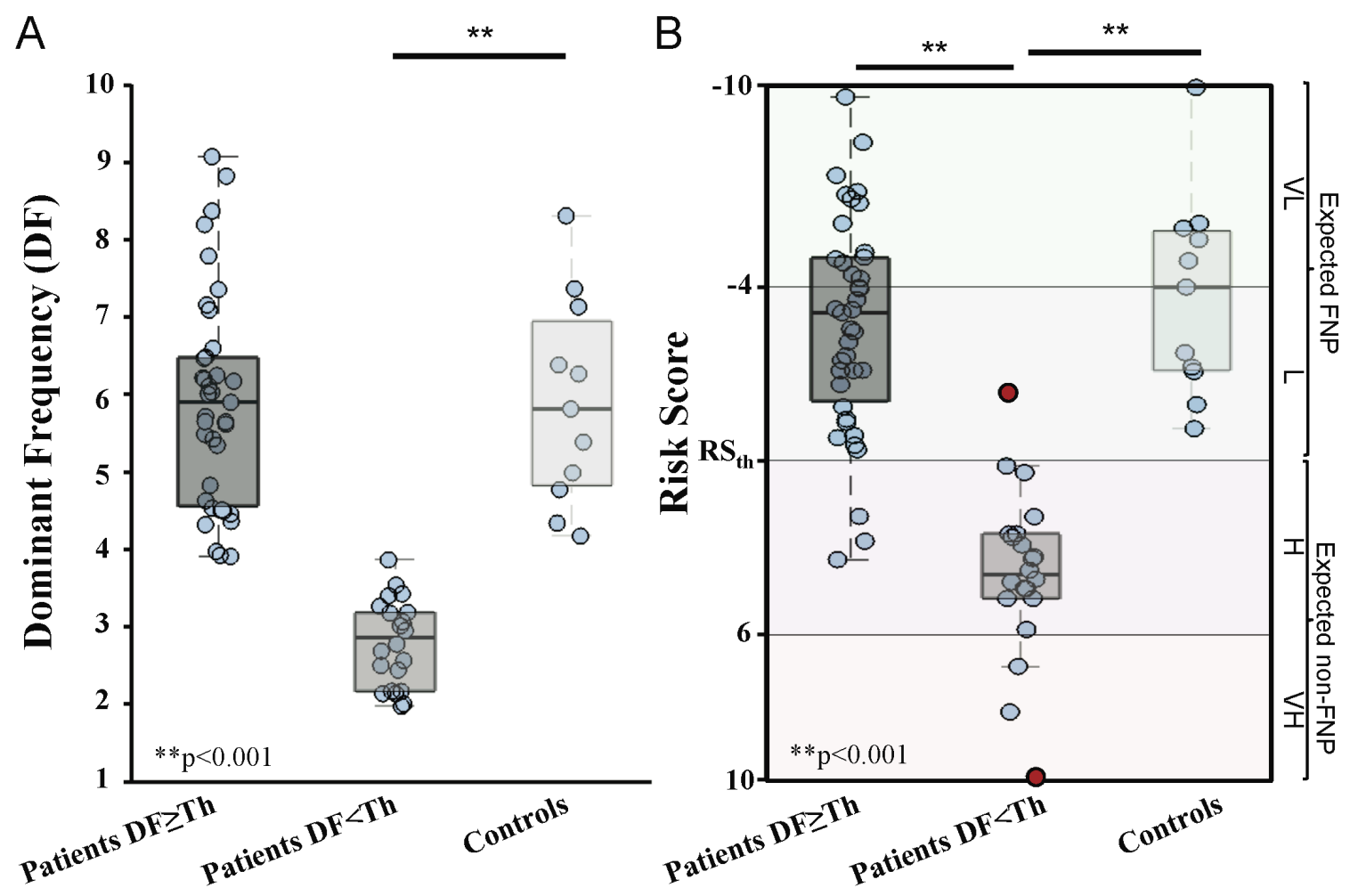
Supplemental Figure 7

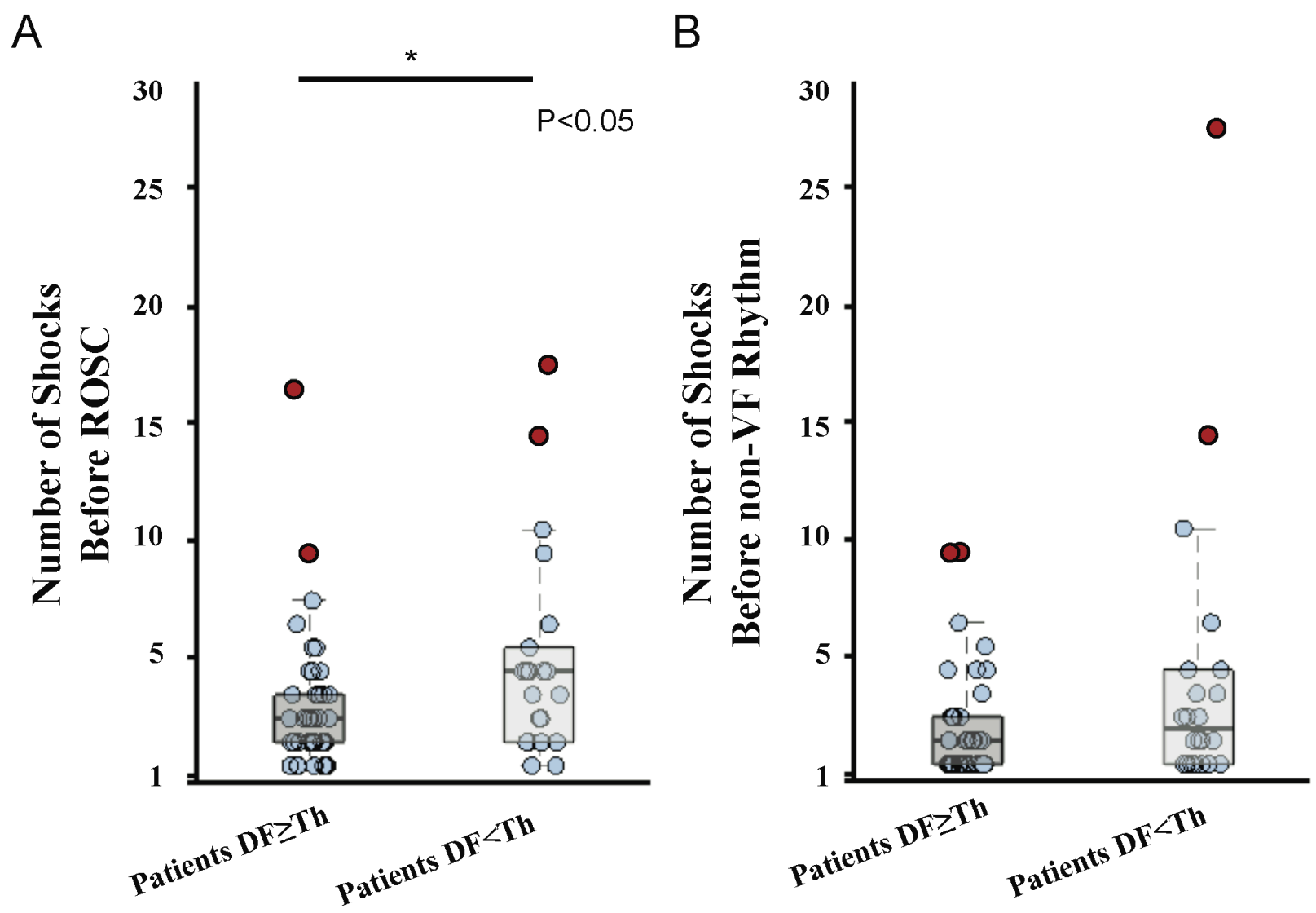


Supplemental Figure 8

A

$$
0.2 \mathrm{mV}
$$

\section{DC-Shock}

$0.5 \mathrm{~s}$

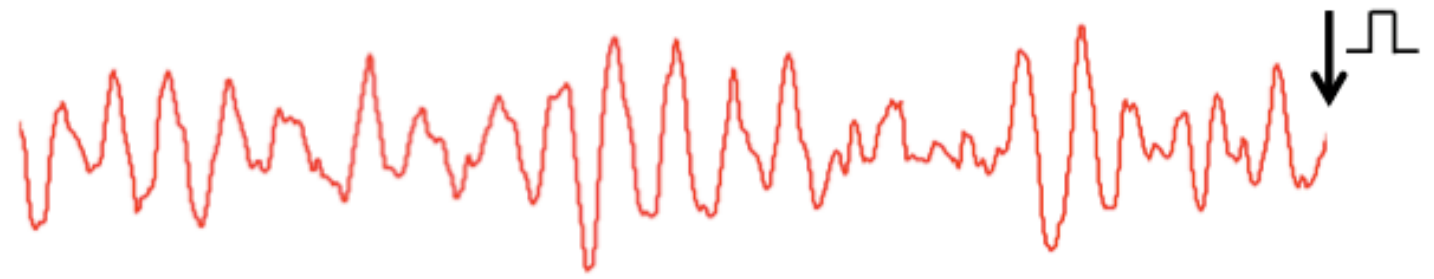

方

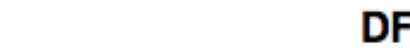

Expected FNP

DF (Hz) HL-pKR HL-PSDR N shocks 3.97

3

2.12

5

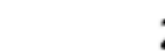

2

34

45

Spectral Frequency $(\mathrm{Hz})$ 
Supplemental Table 1. Glasgow-Pittsburgh Outcome Categorization of Brain Injury

Cerebral Performacnce Categories (CPC)

CPC

1. Good Cerebral Performance: Conscious, alert, and able to work and lead a

normal life. Might have minor psychological or neurological deficits (mild dysphasia, noncapacitating hemiparesis, or minor cranial nerve abnormalities).

2. Moderate Cerebral Disability: Conscious. Sufficient cerebral function for part-time work in a sheltered environment or independent activities of daily life (dress, travel by public transportation, food preparation). Such patients may have hemiplegia, seizures, ataxia, dysarthria, dysphasia, or permanent memory or mental changes.

3. Severe Cerebral Disability: Conscious; patient dependent on others for daily support (in an institution or at home with exceptional family effort) because of impaired brain function. Has at least limited cognition. This category includes a wide range of cerebral abnormalities, from patients who are ambulatory but have severe memory disturbance or dementia precluding independent existence, to those who are paralyzed and can communicate only with their eyes, as in the locked-in syndrome.

4. Coma/vegetative state: Not conscious, unaware of surroundings, no cognition. No verbal and/or psychological interaction with environment.

2

5. Brain Death: Certified brain dead or dead by traditional criteria. 


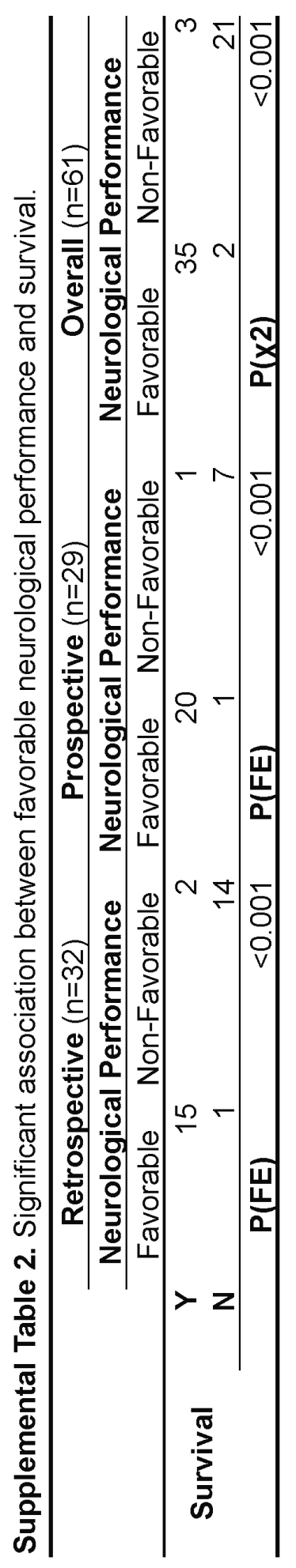


Supplemental Table 3. Follow up performance of the best multivariate predictive model.

Se Sp C-Stat ACC

Favorable Neurological Performance

Retrospective group

$\begin{array}{cccc}1 & 1 & 1 & 1 \\ 0.88 & 0.91 & 0.90 & 0.90\end{array}$

Survival Outcome (Test)

Prospective group

$\begin{array}{llll}0.88 & 0.94 & 0.90 & 0.90\end{array}$

Prospective group

\begin{tabular}{llll}
0.78 & 0.90 & 0.89 & 0.89 \\
\hline
\end{tabular}




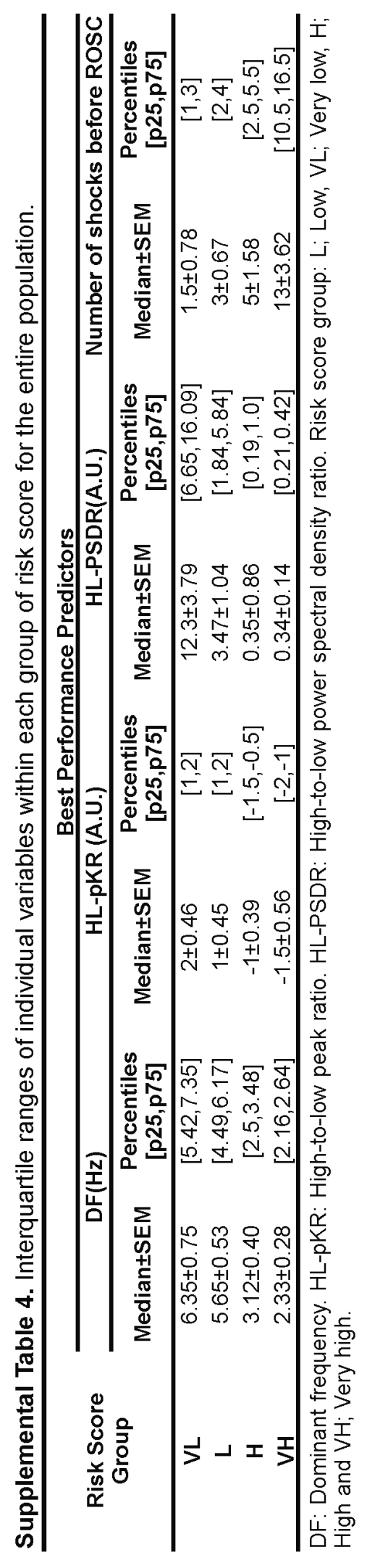




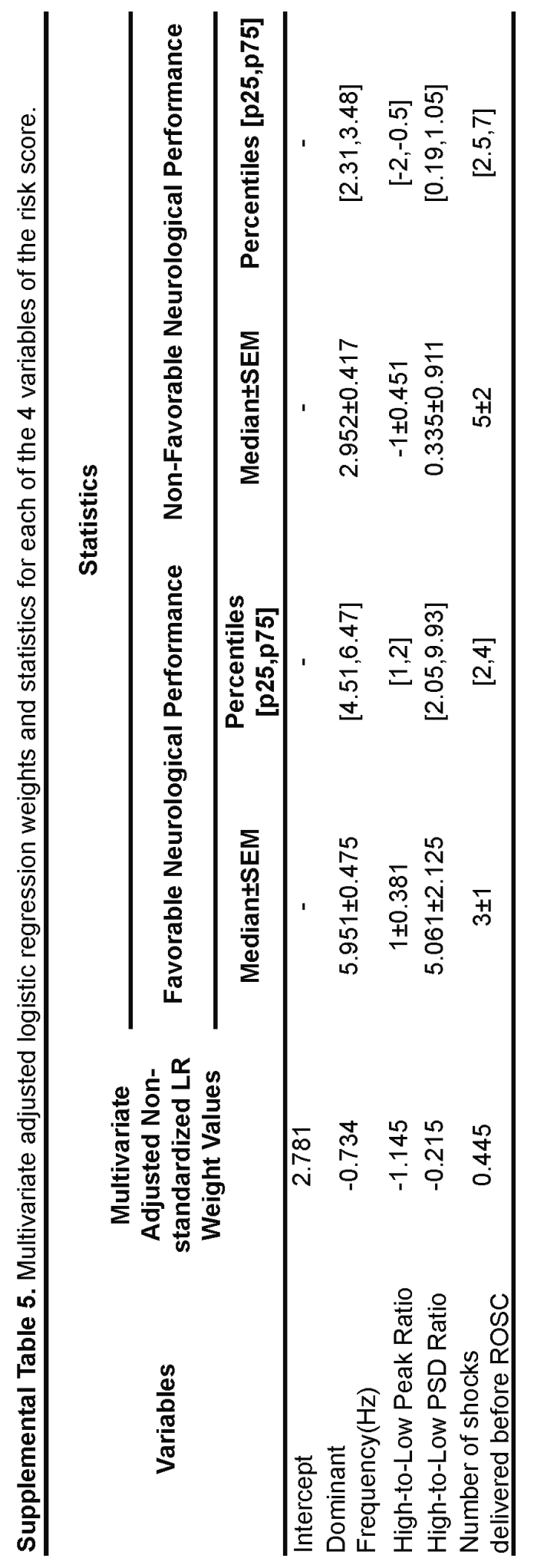


Supplemental Table 6. Contribution of spectral parameters to the predictive performance of the model and comparisons with the best clinical multivariate models.

\begin{tabular}{cc}
\hline Test in & Validation in \\
Retrospective & Prospective \\
Population & Population \\
\hline
\end{tabular}

\begin{tabular}{lcccccc}
\hline & Se & Sp & C-Stat & Se & Sp & C-Stat \\
\hline $\begin{array}{l}\text { I. Best performance multivariate model (4v) } \\
\text { Relative contribution of spectral and clinical predictors }\end{array}$ & 0.94 & 1 & 0.98 & 0.88 & 0.91 & 0.89 \\
II. Spectral prediction model (3v) & & & & & & \\
$\quad$ III. Clinical model (1v) & 0.88 & 0.94 & 0.96 & 0.88 & 0.86 & 0.88 \\
Best clinical models & 0.68 & 0.68 & 0.74 & 0.62 & 0.66 & 0.67 \\
$\quad$ IV. Best clinical model with most associated variables (5v) & & & & & & \\
$\quad$ V. Best clinical model with most associated and relevant variables (8v) & 0.93 & 0.87 & 0.93 & 0.50 & 0.71 & 0.69 \\
\end{tabular}


Supplemental Table 7. Risk score prediction for excluded patients (4 out of 5) due to early mortality.

\begin{tabular}{|c|c|c|c|c|c|c|}
\hline \multirow{2}{*}{ Excluded patients with Early Mortality } & \multicolumn{4}{|c|}{ Predictors } & \multicolumn{2}{|c|}{$\begin{array}{c}\text { Predicted Risk Score } \\
\text { (RS) }\end{array}$} \\
\hline & $\mathrm{DF}(\mathrm{Hz})$ & $\begin{array}{l}\text { HL-pKR } \\
\text { (A.U.) }\end{array}$ & HL-PSD (A.U.) & $\begin{array}{c}\text { Number of shocks } \\
\text { delivered before ROSC }\end{array}$ & RS & RS Group \\
\hline \#1 & 2.16 & -1 & 0.39 & 10 & 6.7 & $\mathrm{VH}$ \\
\hline \#2 & 4.18 & 0 & 1.39 & 1 & -0.14 & $\mathrm{~L}$ \\
\hline \#3 & 4.47 & 1 & 2.77 & 3 & -0.91 & L \\
\hline \#4 & 4.24 & 0 & 1.21 & 1 & -0.15 & L \\
\hline
\end{tabular}

Abbreviations as in Supplemental Table 4. 